\title{
Delirium
}

Jo Ellen Wilson ${ }^{1,2}$, Matthew F. Mart ${ }^{1,3}$, Colm Cunningham ${ }^{4}$, Yahya Shehabi ${ }^{5,6}$, Timothy D. Girard ${ }^{1,7}$, Alasdair M. J. MacLullich ${ }^{8}$, Arjen J. C. Slooter ${ }^{9}$ and

E. Wesley Ely 1,3,10,11

Abstract | Delirium, a syndrome characterized by an acute change in attention, awareness and cognition, is caused by a medical condition that cannot be better explained by a pre-existing neurocognitive disorder. Multiple predisposing factors (for example, pre-existing cognitive impairment) and precipitating factors (for example, urinary tract infection) for delirium have been described, with most patients having both types. Because multiple factors are implicated in the aetiology of delirium, there are likely several neurobiological processes that contribute to delirium pathogenesis, including neuroinflammation, brain vascular dysfunction, altered brain metabolism, neurotransmitter imbalance and impaired neuronal network connectivity. The Diagnostic and Statistical Manual of Mental Disorders, 5th edition (DSM-5) is the most commonly used diagnostic system upon which a reference standard diagnosis is made, although many other delirium screening tools have been developed given the impracticality of using the DSM-5 in many settings. Pharmacological treatments for delirium (such as antipsychotic drugs) are not effective, reflecting substantial gaps in our understanding of its pathophysiology. Currently, the best management strategies are multidomain interventions that focus on treating precipitating conditions, medication review, managing distress, mitigating complications and maintaining engagement to environmental issues. The effective implementation of delirium detection, treatment and prevention strategies remains a major challenge for health-care organizations globally.

The term 'delirium' is derived from the Latin word delirare, meaning 'to go out of the furrow', that is, to deviate from a straight line, to be deranged ${ }^{1}$. Delirium is a severe neuropsychiatric syndrome characterized by the acute onset of deficits in attention and other aspects of cognition. Patients often have altered arousal, from reduced responsiveness at a near-coma level to hypervigilance and severe agitation. They may also experience highly distressing symptoms of psychosis, including delusions and hallucinations, and altered mood. The features of delirium tend to fluctuate in presence and severity. Delirium is associated with considerable distress in patients and caregivers ${ }^{2}$.

Delirium is triggered by multiple potential causes, including acute medical illness, drug use or withdrawal, trauma, or surgery. Most causes originate outside of the brain, but delirium with primary neurological causes, such as stroke, is also recognized. Delirium is variable in duration, with most episodes lasting a few days but with episodes persisting for weeks or months in up to $20 \%$ of individuals ${ }^{3-5}$. The term 'subsyndromal delirium' has been used to describe patients who have some delirium features but do not fulfil all criteria for a delirium diagnosis ${ }^{6,7}$.

Historically, various terms have been used to refer to an acute, global disturbance in mental functioning occurring in the context of medical illness, including encephalopathy, acute brain failure, acute confusional state and organic brain syndrome. The lack of consistent terminology has negatively affected research, with an almost complete segregation of the literature on delirium from that on encephalopathy ${ }^{8}$ despite the manifest overlap in the clinical features of the two syndromes. In addition, clinical communication and coding are adversely affected, with a lack of formal labelling of delirium leading to massive under-representation in hospital discharge data $^{9}$. These issues prompted a consensus statement on nomenclature from an interdisciplinary group, which has been endorsed by ten major professional societies and might help to remove some obstacles to research and clinical care in these interrelated states ${ }^{8}$. The statement advocates using only two terms: delirium and acute encephalopathy. Delirium is defined as the clinical state characterized by a combination of features defined by standard diagnostic systems, such as the Diagnostic and Statistical Manual of Mental Disorders, 5th edition (DSM-5 $)^{4}$. Acute encephalopathy is not a clinical syndrome; rather, it is defined as a rapidly developing (usually within hours to a few days), diffuse pathobiological process that might manifest as delirium or, in cases of severely decreased levels of consciousness, as coma. 


\author{
Author addresses \\ ${ }^{1}$ Center for Critical Illness, Brain Dysfunction, and Survivorship (CIBS), Vanderbilt \\ University Medical Center, Nashville, TN, USA. \\ 2Department of Psychiatry and Behavioral Sciences, Division of General Psychiatry, \\ Vanderbilt University Medical Center, Nashville, TN, USA. \\ ${ }^{3}$ Department of Medicine, Division of Allergy, Pulmonary and Critical Care Medicine, \\ Vanderbilt University Medical Center, Nashville, TN, USA. \\ ${ }^{4}$ School of Biochemistry \& Immunology, Trinity Biomedical Sciences Institute $\&$ Trinity \\ College Institute of Neuroscience, Trinity College Dublin, Dublin, Republic of Ireland. \\ ${ }^{5}$ Monash Health School of Clinical Sciences, Monash University, Melbourne, \\ Victoria, Australia. \\ ${ }^{6}$ Prince of Wales Clinical School of Medicine, University of New South Wales, Sydney, \\ New South Wales, Australia. \\ ${ }^{7}$ Clinical Research, Investigation, and Systems Modeling of Acute Illness Center, \\ Department of Critical Care Medicine, University of Pittsburgh School of Medicine, \\ Pittsburgh, Pennsylvania, USA. \\ ${ }^{8}$ Edinburgh Delirium Research Group, Geriatric Medicine, Usher Institute, \\ University of Edinburgh, Edinburgh, Scotland, UK. \\ ${ }^{9}$ Department of Intensive Care Medicine and UMC Utrecht Brain Center, \\ University Medical Center Utrecht, Utrecht University, Utrecht, Netherlands. \\ ${ }^{10}$ Department of Medicine, Division of General Internal Medicine and Public Health, \\ Vanderbilt University Medical Center, Nashville, TN, USA. \\ ${ }^{11}$ Veteran's Affairs TN Valley, Geriatrics Research, Education and Clinical Center (GRECC), \\ Nashville, TN, USA.
}

Delirium prevalence varies considerably by patient group and setting. Delirium is common in hospitalized older adults in general medical settings, with a 2020 meta-analysis of 33 studies of medical inpatients finding an overall delirium prevalence of $23 \%{ }^{16}$. The prevalence of delirium after surgery ranges from low single-figure percentages in medically well patients undergoing minor elective surgery to $\geq 20 \%$ in high-risk patients undergoing major surgery, especially under emergency conditions ${ }^{17-19}$. For example, in a systematic review of delirium risk in patients undergoing coronary artery bypass grafting, delirium prevalence was $24 \%$ in studies in which a diagnostic instrument was used ${ }^{20}$. The prevalence of postoperative delirium after transcatheter aortic valve replacement was $7.2 \%$ with the transfemoral approach and $21.4 \%$ for other approaches ${ }^{21}$. Delirium is common after acute stroke, with a 2019 systematic review of 32 studies finding a prevalence of $25 \%{ }^{22}$. The prevalence of delirium is also substantial in palliative care settings, with a 2019 systematic review of 42 studies reporting a prevalence of $4-12 \%$ in the community, 9-57\% in patients evaluated in hospital and $6-74 \%$ in inpatient palliative care units, with a pooled prevalence of $35 \% \%^{23}$. Another review found a delirium prevalence of 59-88\% among palliative care inpatients in the weeks approaching death ${ }^{24}$. Fewer data are available about delirium prevalence in community settings, with a prevalence of $4.3-38 \%$ reported in nursing homes ${ }^{25}$, whereas delirium prevalence outside of institutions is likely to be $\leq 2 \%{ }^{26,27}$. Delirium prevalence is high in critical care settings, with a systematic review of studies from North and South America, Europe and Asia reporting a pooled prevalence of $31.8 \%$ in ventilated and non-ventilated intensive care unit (ICU) patients ${ }^{28}$. The prevalence of delirium is generally $50-70 \%$ in mechanically ventilated patients ${ }^{29}$. The wide range of prevalence values of delirium across settings reflects the interaction between the types of patient concerned, who vary in age and frailty, and the number and severity of the precipitating factors.

The prevalence of delirium in infants, children and adolescents is less well understood. Studies from Europe and the USA report prevalence estimates of delirium in children and adolescents ranging from $4 \%$ to almost $50 \%$ in critically ill children and adolescent $\mathrm{s}^{30-35}$. In one study from the USA, more than half of infants under 2 years of age experienced delirium while critically ill ${ }^{36}$. The prevalence of subsyndromal delirium is highly variable and depends on the population being studied and which delirium definition is used. However, in one study from North America, almost two-thirds of adults over 65 years of age who were admitted to an inpatient medical or geriatric service had subsyndromal delirium ${ }^{7}$.

\section{Risk factors}

The risk of delirium is determined by predisposing risk factors (that is, the background characteristics of patients) and precipitating risk factors (that is, acute insults, injury or drugs). Predisposing risk factors for delirium include increased age, cognitive impairment (such as dementia ${ }^{37}$ or developmental delay ${ }^{38}$ ), frailty, comorbidities (including cardiovascular and renal disease), depression or other psychiatric illness ${ }^{39,40}$, alcohol 


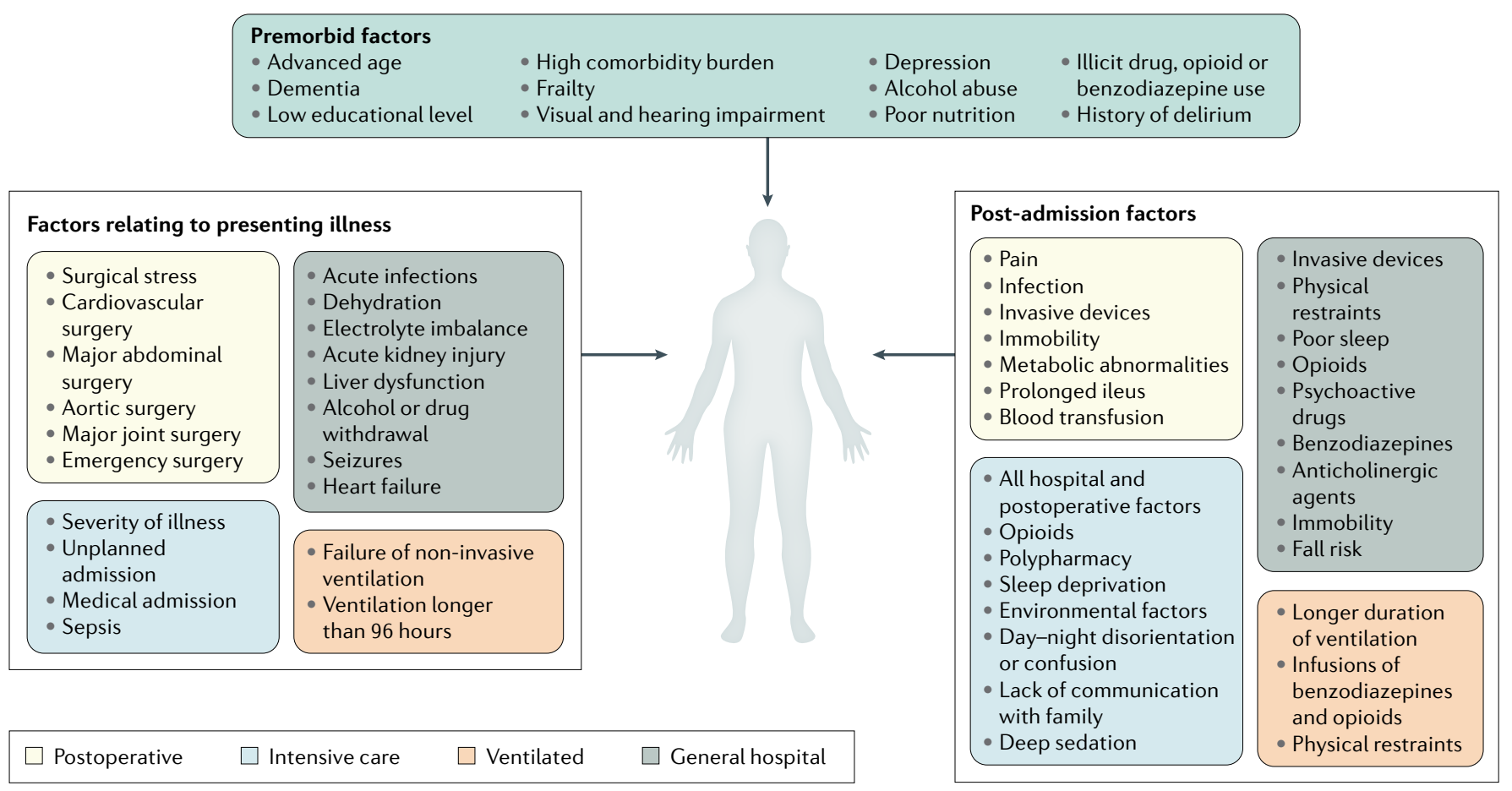

Fig. 1 | Risk factors for delirium. Risk factors for delirium relate to premorbid or predisposing factors (that is, a patient's characteristics) and to precipitating factors, which are factors relating to the presenting illness or that occur after hospital or intensive care unit admission.

use, poor nutritional status ${ }^{41,42}$, and visual and hearing impairment ${ }^{18,27}$. Total risk depends on the number of risk factors in each individual and, where applicable, their severity; for example, frailty, which typically encompasses a number of risk factors, is strongly associated with delirium risk ${ }^{43,44}$, and the degree of cognitive impairment shows a strong linear association with delirium risk ${ }^{45}$. Furthermore, neuroimaging studies indicate that the risk of delirium might be higher in individuals with greater cerebral atrophy and/or greater white matter disease ${ }^{46,47}$. Genetic studies have not identified consistent candidate genes associated with delirium risk but these studies are few and underpowered ${ }^{48,49}$.

Precipitating factors for delirium span a wide range of different kinds of insults, including, amongst others, acute medical illness (such as sepsis, hypoglycaemia, stroke and liver failure), trauma (such as fractures or head injury), surgery, dehydration and psychological stress $^{17,27}$. Typically, more than one precipitating factor is present in patients ${ }^{50,51}$. In addition, drug use and withdrawal and medication changes are associated with delirium. Of note, benzodiazepines, dihydropyridines (L-type calcium channel blockers typically used in the treatment of hypertension), antihistamines and opioids may convey the highest risk of delirium, although insufficiently managed pain may itself be a risk factor ${ }^{52,53}$; however, the exact relationship between pain medication, pain management and delirium risk remains unclear. In addition to common premorbid factors, specific health-care setting-related factors, such as mechanical ventilation ${ }^{54-59}$, are risk factors for hospital-acquired delirium (FIG. 1). Many of these factors may coexist in different health-care settings.

\section{Prediction models}

Risk factors for delirium have been combined in delirium prediction models in different populations, such as in patients who have undergone cardiac or orthopaedic surgery or in older patients $(\geq 65 \text { years of age })^{60-66}$. However, existing delirium prediction models use different methods of delirium identification and different risk factors for model calibration ${ }^{67}$. A 2018 systematic review of prediction models for older adult inpatients in general settings concluded that existing models do not have adequate predictive capabilities ${ }^{60}$. In the ICU, the PRE-DELIRIC model ${ }^{68}$, which is based on ten risk factors (age, APACHE-II score, admission group, coma, infection, metabolic acidosis, use of sedatives and morphine, urea concentration and urgent admission) identified within 24 hours of ICU admission, predicted delirium with an area under the receiver operating curve of 0.87 (95\% CI $0.85-0.89$ ) in the first 24 hours after admission to the ICU. The PRE-DELIRIC model showed excellent performance in clinical cohorts, including in studies from Lithuania, Scotland and Argentina ${ }^{69-71}$.

\section{Outcomes}

The natural history of delirium is characterized by heterogeneity ${ }^{72}$. The duration of delirium can vary widely, with delirium lasting a few days in most patients yet for weeks or months in many others. Persistent delirium (that is, lasting for weeks or months) is not rare, with $20 \%$ of patients exhibiting some symptoms of delirium at 6 months $^{73}$. Delirium also varies considerably in the features of the syndrome that are present and in their severity, as judged by parameters such as the level of impairment of neuropsychological domains 
(orientation, level of arousal, attention, memory, comprehension and vigilance), psychotic features and mood disturbance, and duration ${ }^{74}$. Delirium is independently associated with multiple poor outcomes, including increased post-discharge mortality as well as new institutionalization and dementia ${ }^{75}$. Features of delirium that predict worse outcomes include older age, frailty, hypoactive subtype, delirium duration and delirium severity $^{76}$. Persistent delirium, even with partial recovery, is strongly associated with poor outcomes ${ }^{5}$.

Delirium is a well-established risk factor for both incident dementia and worsening of existing dementia. For example, in a large, 10-year prospective population study of people $\geq 85$ years of age, delirium was strongly associated with incident dementia (OR 8.7; 95\% CI 2.1-35) and with acceleration of dementia severity (OR 3.1; 95\% CI 1.5-6.3 $)^{77}$. The association between delirium and risk for long-term cognitive decline in both medical and surgical populations was confirmed in a meta-analysis of 23 studies, which reported an estimated effect size (Hedges' g) of 0.45 (95\% CI 0.34-0.57) ${ }^{78}$.

\section{Mechanisms/pathophysiology Framing delirium pathogenesis research}

As several different factors are implicated in the aetiology of delirium, including sepsis, fractures, surgery, medication changes, hypoglycaemia and liver failure, it follows that distinct neurobiological mechanisms or combinations of them are involved in delirium pathogenesis. Unravelling these mechanisms in different patient populations and clinical settings is necessary. However, given that multiple aetiological factors may contribute to delirium in a patient, several neurobiological mechanisms may also interact to produce the observed syndrome, and it remains difficult to classify delirium based either on distinct aetiologies or on distinct neurobiological mechanisms. Nonetheless, delirium with a single aetiology (that is, involving a single causative factor) has been demonstrated in human studies of hypoxia, hypoglycaemia ${ }^{79}$ and cholinergic antagonism ${ }^{80}$. As the different aetiologies lead to a shared, albeit heterogeneous, core syndrome, some researchers have proposed that a common pathogenetic pathway underpins delirium; however, evidence is currently lacking for such a single pathway. Although adopting the term 'delirium' has been valuable in highlighting its importance for patient outcomes, 'lumping' together all types of delirium when researching the underlying neurobiology of the disorder might be less useful than 'splitting' the syndrome into different subtypes based on aetiological contributors (that is, the underlying acute pathophysiological disruption or precipitants leading to delirium $)^{10}$.

In clinical studies, disentangling the contributions of multiple triggers and processes, such as hypoxia, inflammation and sedation, is possible but only to a limited extent $^{81}$. Thus, studies in animal models of relatively 'pure' aetiologies are important to allow strong predictions to be made about which biological factors have a causative role in exceeding key thresholds and triggering delirium. Nonetheless, it remains important to seek convergence between deliria of different aetiologies and we adopt both approaches here.
As it is difficult to incontrovertibly demonstrate the presence of delirium in experimental animals such as mice and rats, one has to be pragmatic: the aim of animal models for delirium research must be to demonstrate deficits in brain function that resemble symptoms observed in patients with delirium (face validity) and these deficits should arise from experimental manipulations that resemble the clinical insults (such as sepsis, fracture and infection) that produce delirium in humans (construct validity). If these criteria are satisfied, then animal models can be useful for elucidating which cellular and molecular changes might have a causal role in inducing the observed brain dysfunction. In this section, we aim to integrate data from human and animal model studies to create an evidence-based conceptual framework of delirium pathophysiology.

\section{Degenerating brain vulnerability}

The interaction between an underlying predisposition and a superimposed acute stressor is key to delirium pathophysiology. Although major acute stressors (such as head injury, stroke and septic shock) can trigger delirium, even in resilient individuals, predisposing factors (such as old age, frailty and existing cognitive impairment) also substantially increase delirium risk ${ }^{43,82}$. Prior cognitive impairment is a progressive risk factor: as baseline cognition decreases, delirium risk increases in a linear fashion ${ }^{45}$ and, therefore, in both rodents and humans, less-severe acute stressors (such as surgery or infections) are sufficient to trigger delirium.

In higher-risk individuals, delirium is a failure of the vulnerable brain to show resilience in response to an acute stressor. This vulnerability can be caused by a multitude of processes that are not mutually exclusive. Key processes include changes in brain connectivity, neuroinflammatory and glial cell alterations, and vascular changes. First, brain network connectivity is impaired by ageing and neurodegeneration ${ }^{83}$ and cholinergic and noradrenergic neuronal populations degenerate with increasing age and dementia, both of which have consequences for network and cognitive function in response to acute stressors. Second, animal model studies show that both microglia and astrocytes are 'primed' by existing neurodegeneration to produce exaggerated pro-inflammatory responses to secondary inflammatory stimuli, thereby exacerbating inflammation specifically in areas made vulnerable by neurodegeneration ${ }^{84,85}$. Furthermore, astrocytes are metabolically impaired by losing interactions with healthy neurons during neurodegeneration, further undermining their support for neuronal metabolism ${ }^{86}$. Third, ageing and neurodegeneration also trigger alterations in the brain vasculature ${ }^{87}$. These vascular changes lead to impaired brain perfusion and vascular reactivity, disruption of the transport of important plasma proteins into the brain ${ }^{88}$ and leakiness of the blood-brain barrier (BBB), potentially making the brain more vulnerable to disruptions of energy or oxygen supply and the effects of circulating inflammatory molecules.

This list is not exhaustive and the potential for acute stress to disrupt function in the vulnerable brain can be further potentiated by poor nutrition and hydration 


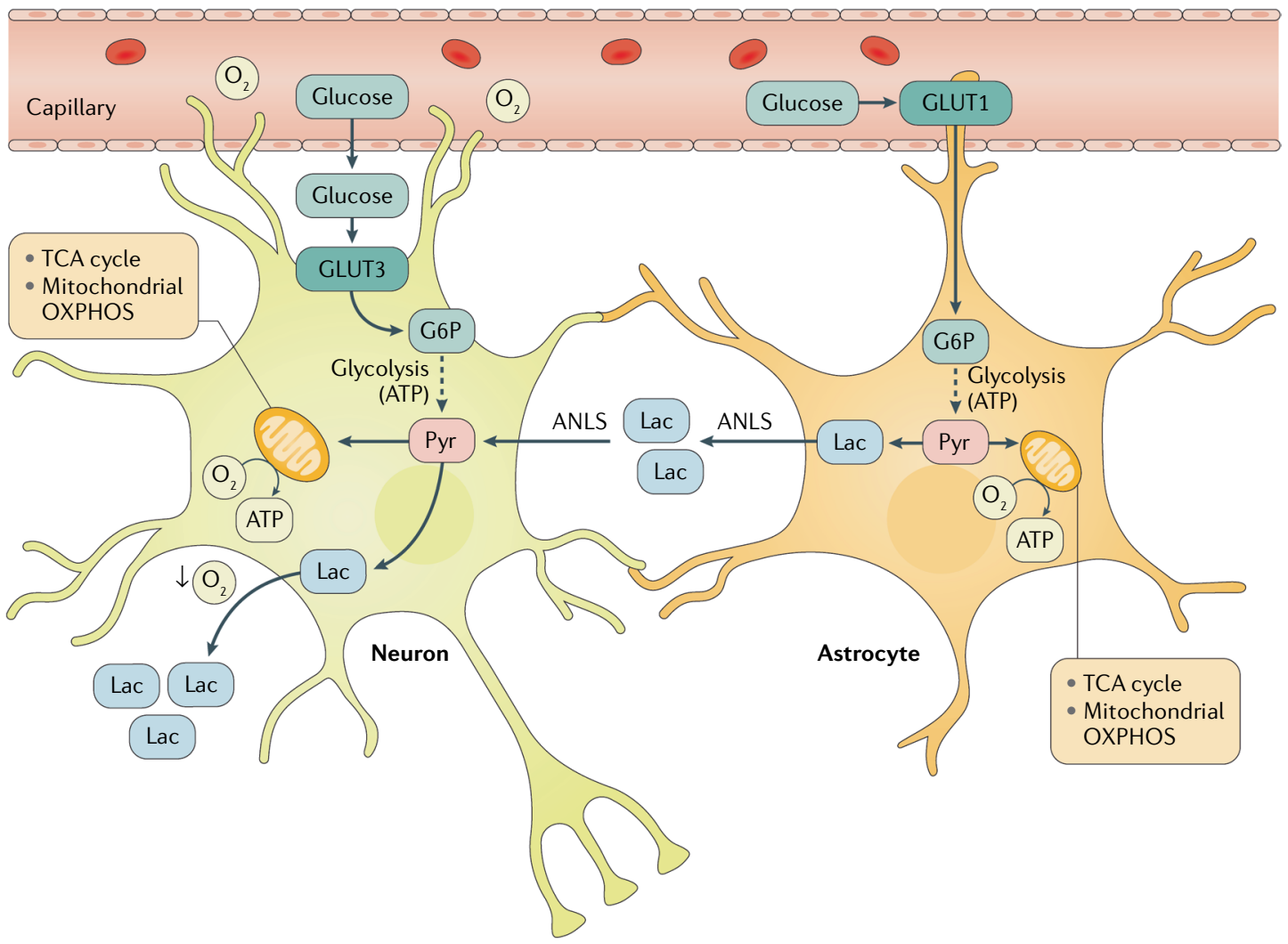

Fig. 2 | Bioenergetic insufficiency may underpin delirium in multiple scenarios. Neurons and astrocytes both use glucose supplied by the microvasculature to generate ATP by glycolysis. In addition, in the astrocyte-neuron lactate shuttle (ANLS), lactate (Lac) synthesized by astrocytes after glycolysis can be exported for use by neurons, which convert Lac into pyruvate (Pyr) that is imported into the mitochondria and used to fuel the tricarboxylic acid (TCA) cycle. There are many ways in which the brain or regions of the brain may become dysfunctional owing to energy insufficiency, and there is support for the idea that these may contribute to the precipitation of delirium. First, respiratory distress produces hypoxaemia and can cause brain hypoxia, limiting neuronal energy metabolism. That is, in hypoxic conditions, insufficient oxygen $\left(\mathrm{O}_{2}\right)$ supply leads to impaired mitochondrial oxidative phosphorylation (OXPHOS) and insufficient generation of energy, in the form of ATP. In these conditions, glycolysis-generated Pyr, instead of being imported into the mitochondria, forms excess Lac, which can be measured in the extracellular fluid. Second, septic shock reduces blood flow, producing both hypoxia and impaired glucose supply. Third, even with adequate systemic blood flow, brain microcapillary dysfunction may produce brain tissue hypoxia and neuroglycopenia. Fourth, even with normal blood pressure, if neurovascular coupling is impaired, vessels may fail to meet the specific demands of regional neuronal activity and thereby block higher-order brain functions. Fifth, systemic hypoglycaemia can lead to insufficient brain glucose supply, delirium and coma. Sixth, even with adequate delivery of glucose to the brain, insulin resistance may result in impaired glucose utilization (not shown). Seventh, altered expression of glucose transporters (GLUT1 and GLUT3), for example, in the degenerating brain, may limit glucose uptake by the endothelium, astrocytes or neurons, thereby limiting the glucose-6-phosphate (G6P) required for glycolysis and limiting the generation of Pyr required for the TCA cycle. Last, impairment of astrocyte function may limit their ability to release glycogen from intracellular stores, metabolize glucose and provide Lac to neurons for energy metabolism.

and by ageing-associated renal and hepatic impairment, causing slower metabolism and clearance of potentially neurotoxic drugs and metabolites ${ }^{89}$. Furthermore, impaired BBB function may increase the permeability of systemically administered medications that can have psychoactive and deleterious effects on brain function. Therefore, acute derangements occurring during acute illness or trauma have greater effects on a vulnerable brain than on a young healthy brain.

\section{Mechanisms precipitating delirium}

As mentioned earlier, delirium is triggered by a wide variety of acute medical conditions. Focusing on potential biological mechanisms rather than on the specific medical events that may trigger them, we aim to highlight the overlap in molecular pathways between deliria with different aetiologies and to identify mechanisms of convergence.

Brain energy metabolism. The brain requires a large amount of energy and, as either oxygen or glucose deficiency can markedly constrain brain function, acute illness might impair brain metabolism in multiple ways (FIG. 2). The long-standing 'cerebral metabolic insufficiency ${ }^{90}$ hypothesis proposes that delirium is caused by the failure to meet the energy requirements of the brain and, although this hypothesis is supported by data from various sources, as we discuss below, substantial gaps in our understanding persist owing to the paucity of studies directly testing this hypothesis in animals or patients. 


\section{Box 1 | Delirium and long-term cognitive sequelae}

Episodes of delirium have long-term cognitive consequences. For example, cognitive dysfunction after intensive care unit delirium was similar to the cognitive deficits seen with moderate traumatic brain injury ${ }^{3}$, delirium in the context of Alzheimer disease was associated with an acceleration of existing dementia ${ }^{323}$ and delirium in aged populations was associated with an eightfold increased risk of subsequent dementia ${ }^{77}$. Dementia after delirium might be associated with new pathological signatures rather than with exacerbation of amyloid- $\beta$ and tau pathology, consistent with a new brain injury occurring during delirium ${ }^{77}$. This idea is supported by emerging clinical studies showing elevation of the axonal injury marker neurofilament light chain in medical and surgical patients with delirium ${ }^{355-357}$, markers of synaptic damage in patients with delirium after infection ${ }^{188}$ and the finding of persistent cognitive deficits and hippocampal atrophy in intensive care unit patients with delirium at 6-24 months after discharge ${ }^{358}$. Animal model studies previously demonstrated new neuronal death ${ }^{84}$ and tau and synaptic pathology $\mathrm{y}^{359}$ after systemic lipopolysaccharide administration and surgical challenges in animals with prior neurodegenerative disease.

It is not clear whether delirium drives new brain injury or whether acute medical or surgical events trigger delirium and acute brain injury by parallel pathways. Delirium may simply signal the severity of current brain disruption rather than actually causing injury. Rodent studies show that electrophysiological hyperexcitability and neuronal death induced by systemic lipopolysaccharide administration are dependent on IL-1 receptor type 1 but are mechanistically distinct from the acute cognitive dysfunction that is also triggered by similar lipopolysaccharide challenges ${ }^{129}$. These data suggest that delirium and acute brain injury may occur by distinct mechanisms, which could mean that successfully treating one may not limit the other. Similarly, there is evidence for coagulopathy and vascular injury during sepsis ${ }^{98}$ and SARS-CoV- 2 infection ${ }^{146}$, both of which produce a systemic inflammatory cytokine response and frequently lead to delirium. Therefore, it is essential to clarify the temporal and causal relationships between inflammation, hypoxia, delirium and injury in multiple acute illness settings.

Effective blood oxygenation is essential to avoid brain sequelae. As several clinical scenarios can lead to hypoxia, this is a highly plausible driver of delirium. For example, impaired lung function during respiratory distress causes hypoxaemia, haemodynamic shock can impair blood flow to the brain and, even after blood pressure normalization, microcirculatory impairment can still result in decreased brain perfusion and tissue hypoxia ${ }^{91}$. In small studies in patients, delirium was associated with decreased cerebral blood flow (30-50\%) that resolved at the time of recovery ${ }^{92}$ and with impaired autoregulation in patients with sepsis ${ }^{93}$. Consistent with a possible role for hypoxia, cerebrospinal fluid (CSF) lactate levels were significantly elevated in hip fracture and general medical patients with delirium ${ }^{94,95}$. Brain tissue hypoxia can be assessed in patients using near infrared spectroscopy. A systematic review found evidence for an association between low regional cerebral oxygenation and delirium, although there are still too few studies for a meta-analysis ${ }^{96}$.

Although animal studies of brain hypoxia have not addressed delirium explicitly, lipopolysaccharidetriggered microcirculatory dysfunction in rodents precedes decreased brain oxygenation and impaired neurophysiological function as measured by a loss of evoked potential $s^{97}$, demonstrating a plausible temporal link between brain hypoxia and disruption of brain function. Furthermore, experimental hypoxia in human volunteers triggers electroencephalography (EEG) slowing, which directly preceded the disruption of attention, awareness and comprehension (effectively, experimental delirium); this data probably represents the clearest evidence that hypoxia, on its own, is sufficient to directly cause delirium ${ }^{90}$. Hypercoagulation, such as that widely reported during SARS-CoV-2 infection, may also contribute to brain hypoxia (discussed below).

Gross ischaemia shows an association with delirium but, even in a cohort of patients with septic shock who experienced delirium, only 12 of 31 patients showed MRI evidence of ischaemic stroke or white-matter ischaemic lesions ${ }^{98}$. These MRI changes were measured at a median of 3 days after the onset of clinical signs of delirium and were associated with long-term adverse outcomes, and indeed might be more relevant to post-delirium brain injury (BOX 1).

In addition to gross changes in blood flow and blood oxygenation, there is evidence for impaired neurovascular coupling in ageing and dementia ${ }^{87}$. Thus, even when brain perfusion is normal, dynamic increases in regional brain activity may not be adequately supported by functional hyperaemia, meaning that activity-dependent increases in oxygen and glucose cannot be achieved in specific brain regions when required, with likely consequences for brain functions requiring this dynamically increased flow. Finally, even in normoxia, mitochondrial dysfunction, as occurs in dementia ${ }^{99}$, might impair the utilization of pyruvate and other substrates for ATP generation.

Constrained supply or impaired use of energy substrates may also contribute to delirium. Hypoglycaemia is sufficient to produce EEG slowing and delirium in volunteers ${ }^{90}$, in patients with iatrogenic insulin-induced hypoglycaemia ${ }^{100}$ and in diabetes mellitus ${ }^{101}$. More severe, prolonged hypoglycaemia can lead to generalized delta in EEG recordings, contributing to unreactive $E G^{102}$. Hypoglycaemia also substantially increases the risk of delirium in ICU patients ${ }^{103}$. In mice, both lipopolysaccharide and IL- $1 \beta$ trigger hypoglycaemia, selectively causing acute cognitive dysfunction in animals with prior neurodegeneration, and glucose treatment mitigated these cognitive deficits ${ }^{95}$. Hypoglycaemia is not commonly observed in patients with delirium but infection, surgery and trauma can all trigger insulin insensitivity ${ }^{104,105}$, which limits glucose uptake and use. Glucose utilization ${ }^{106}$ and insulin signalling ${ }^{107}$ are markedly reduced in patients with Alzheimer disease and these individuals might, therefore, be particularly susceptible to the effects of limited glucose availability, as was shown in an animal model of delirium ${ }^{95}$. FDG-PET imaging data revealed that glucose uptake is substantially reduced in rats with lipopolysaccharide-induced sepsis ${ }^{108}$ and human FDG-PET imaging data showed reduced glucose metabolism during delirium ${ }^{109}$. Thus, reduced brain glucose utilization may be driven by decreased blood glucose, brain insulin insensitivity or impaired glucose uptake by endothelial cells or astrocytes through the glucose transporter GLUT1, the expression of which is reduced in models of Alzheimer disease $^{87}$. Indeed, astrocytes also support brain energy metabolism by glycogen mobilization and by the functioning of the astrocyte-neuron lactate shunt (FIG. 2), with the clinical FDG-PET signal at least partly accounted for by astrocyte glucose metabolism ${ }^{110}$. Thus, acute illness can alter the availability of energy substrates, and ageing-dependent or neurodegeneration-dependent 
impairment of astrocyte neuronal support ${ }^{86}$ might also contribute to cerebral metabolic insufficiency and, potentially, to delirium.

In conclusion, the cerebral metabolic insufficiency hypothesis ${ }^{90}$ remains a plausible explanation for delirium in several scenarios (FIG. 2). However, substantial research effort is now needed to confirm a direct causative role of cerebral metabolic insufficiency in delirium and to move from simply providing support for this hypothesis to using this information to identify targets for therapeutic intervention.

Inflammation. Peripheral inflammation is a wellestablished trigger of delirium ${ }^{111}$, but the precise mechanisms by which it disrupts brain function are not clearly understood and most of the discussion here relates to animal model studies (FIG. 3, FIG. 4). Although many of these findings require validation in patients, it is clear that inflammation induces pathways that are activated during delirium in many clinical settings.

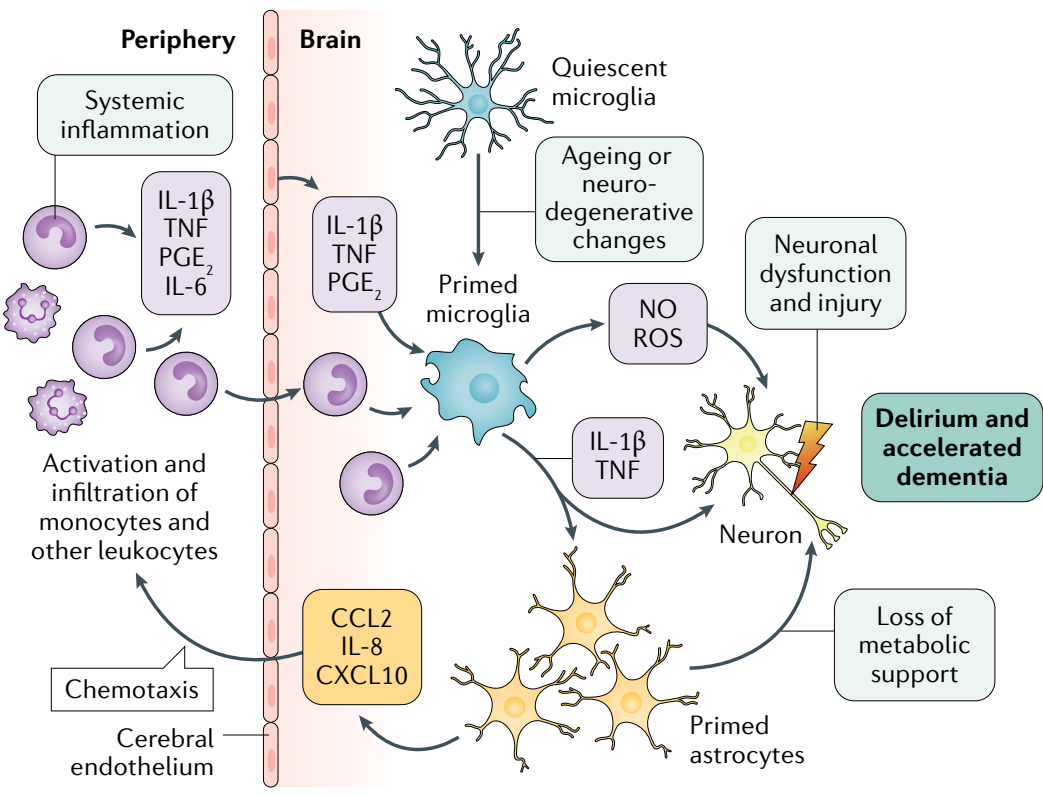

Fig. 3 | Inflammatory mechanisms in delirium. Inflammatory trauma, surgery, infection and sepsis can trigger delirium. These diverse stressors may share pathogenetic mechanisms, including increased local and circulating levels of damage-associated molecular patterns (in surgery and trauma) and pathogen-associated molecular patterns (in infection and sepsis). These stimuli trigger tissue macrophage and blood monocyte activation and secretion of inflammatory mediators, such as IL-1, IL-1 $\beta$, IL-6, tumour necrosis factor (TNF) and prostaglandin $\mathrm{E}_{2}\left(\mathrm{PGE}_{2}\right)$. These molecules may, to a limited extent, cross the blood-brain barrier but their production is also induced in the brain endothelium and epithelium (not shown) and secreted directly into the brain parenchyma by endothelial, epithelial and brain perivascular macrophages. By mechanisms that are not entirely clear, microglia are then triggered to produce pro-inflammatory cytokines, reactive oxygen species (ROS) and reactive nitrogen species. If primed by prior pathology in the brain (such as amyloid or prior neurodegeneration), these microglia produce increased levels of these mediators, which affect both astrocytes and neurons. Cytokine-stimulated astrocytes produce increased levels of chemokines, contributing to the recruitment of monocytes and other immune cell populations to the brain, but their activation also leads to a loss of metabolic support for neuronal energy metabolism. Microglial-derived inflammatory mediators, such as IL-1 $\beta$ and TNF, directly affect neuronal function to produce both dysfunction and injury or cell death, which might collectively contribute to acute behavioural manifestations in the delirium syndrome but also produce new brain injury that promotes long-term cognitive decline. NO, nitric oxide.
Although orthopaedic fracture and sepsis populations differ, they share key inflammatory pathways, including the activation of Toll-like receptor 4 (TLR4) on tissue macrophages by pathogen-associated molecular patterns (such as lipopolysaccharide) or by damage-associated molecular patterns (such as HMGB1) and the release of inflammatory mediators such as IL- $1 \beta$, tumour necrosis factor (TNF) and chemokines at the site of insult ${ }^{112}$. Human studies show that anaesthesia alone (in the absence of the inflammatory trauma of surgery) is not a contributor to these inflammatory changes ${ }^{113}$. Animal studies show that these mediators and circulating leukocytes activate the brain by multiple routes ${ }^{114}$, but it is clear that microbial products, inflammatory mediators and leukocytes also interact directly with the brain vasculature to stimulate cytokine, chemokine and prostaglandin secretion from brain endothelium and perivascular macrophages ${ }^{115,116}$ (FIG. 3, FIG. 4). Circulating mediators may also enter the brain parenchyma, albeit to a limited extent ${ }^{117}$.

Although neuroinflammatory hypotheses of delirium are widely discussed ${ }^{111,18,119}$, most clinical delirium data address an imprecisely specified inflammatory hypothesis rather than a specifically neuro-inflammatory hypothesis. Clearly, severe systemic inflammation, as measured by circulating cytokines (such as IL-6, TNF and MCP1) or acute-phase proteins (such as CRP), is associated with increased delirium risk in adults undergoing major surgery or who are critically ill ${ }^{120-122}$. However, these mediators are robustly induced in all patients with sepsis, trauma or surgery and not only in those who develop delirium. Therefore, prior brain vulnerabilities likely remain key determinants of whether such inflammation actually triggers delirium.

Microglial activation has been associated with delirium in a small post-mortem study including patients who died of shock and respiratory insufficiency ${ }^{123,124}$. Furthermore, studies in mice showed that microglia are necessary to mediate postoperative cognitive dysfunction in mice ${ }^{125}$ and that they become primed by neurodegeneration to show exaggerated IL-1 synthesis in response to acute systemic inflammation ${ }^{84}$. In rodent studies, the disruption of behavioural function by lipopolysaccharide-induced sepsis, Escherichia coli infection or trauma was mediated by IL-1 (REFS ${ }^{126-128}$ ) and experimentally administered IL- $1 \beta$ disrupted neuronal function selectively in the degenerating brain ${ }^{129}$. An association of elevated plasma IL-1 with encephalopathy in patients with sepsis ${ }^{129-131}$ and of elevated CSF IL- $1 \beta$ with delirium in hip fracture patients ${ }^{130}$ provides support for a possible causative effect of IL-1 in delirium.

IL-1 acts directly on multiple brain cell types ${ }^{132}$. Acute administration of IL- $1 \beta$ and TNF activates astrocytes to produce chemokines (such as CCL2, CXCL1 and CXCL10) and this is also exaggerated in neurodegeneration ${ }^{85,133}$, facilitating the homing of activated leukocytes to the brain. Increased monocyte and neutrophil recruitment contributes to cognitive dysfunction and/or brain damage in mouse models of sepsis, viral encephalitis and postoperative cognitive function ${ }^{134-136}$ but the mechanisms are poorly understood. Increased circulating or CSF levels of several 


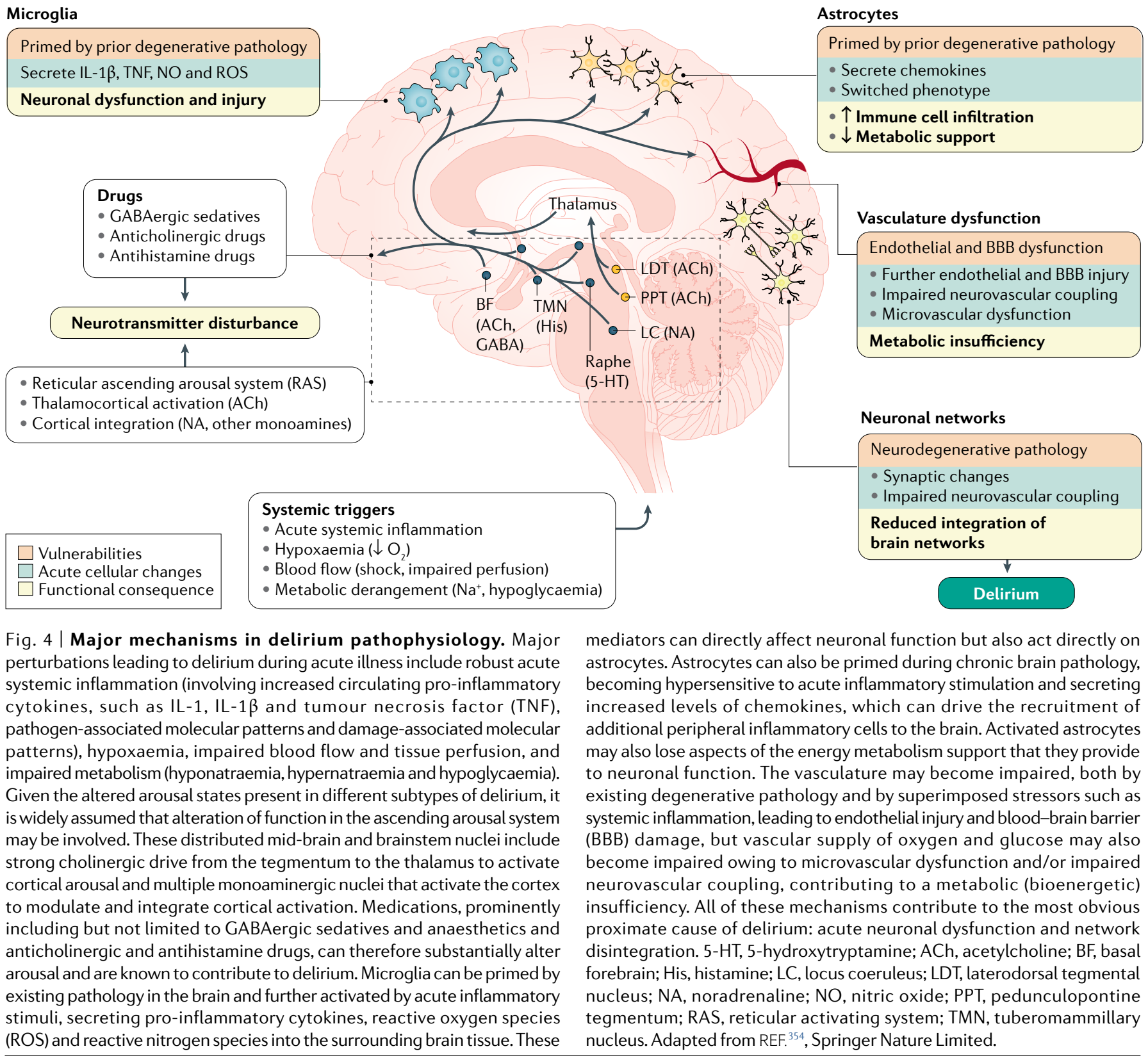

chemokines, including IL-8 and CCL2, are associated with human delirium ${ }^{120,137-140}$ and, as these molecules can also act directly on several neuronal populations ${ }^{141-143}$, their contribution to disrupting functional connectivity in neuronal networks relevant to delirium merits investigation. IL-1 $\beta$, TNF and other inflammatory mediators also activate the vasculature, facilitating leukocyte infiltration and dynamically controlling BBB permeability ${ }^{144}$. Although BBB disruption is widely assumed to be pivotal in inflammation-induced delirium, no direct evidence exists to support this assumption. Although an elevated CSF level of albumin (a marker of increased permeability of the blood-CSF barrier) is associated with delirium in hip fracture patients, blood-CSF barrier permeability was not increased in most patients with delirium ${ }^{145}$. Therefore, despite its plausibility, a causative role for increased BBB or blood-CSF barrier permeability in delirium remains to be shown and verifying their contribution is an important research priority.

Inflammation may also contribute to delirium through the promotion of coagulation, which is common in sepsis and, more recently, in SARS-CoV-2 infection $^{146}$. Hypercoagulation correlates with reduced levels of activated protein $\mathrm{C}$ or increases in D-dimer and can produce effects ranging from impaired cerebral autoregulation $^{93}$ to thrombosis and ischaemia. Although it is clear that acute inflammation can trigger delirium, studies tracking inflammatory changes before, during and after delirium in humans are limited ${ }^{147}$ and considerable effort in human and animal studies is required to dissect exactly which molecules act on which brain regions to trigger which type of dysfunction. Current knowledge has been elucidated predominantly from animal models (FIG. 4). 
Drugs, stress and neurotransmitter imbalance. Drug use and medication changes can precipitate delirium, sometimes by direct effects on brain neurotransmitter systems, such as acetylcholine (ACh), dopamine and GABA, whereas polypharmacy and impaired renal and hepatic function may lead to inappropriately high or prolonged blood drug concentrations and adverse drug interactions ${ }^{89}$. Although experimentally disrupting function in multiple neurotransmitter systems in animals can produce some features of delirium, these studies have rarely been performed with delirium as an explicit outcome. Similarly, studies of neurotransmitter levels in the CSF during delirium have not revealed clear changes in levels of these molecules ${ }^{139}$. However, using information on drug-induced delirium in humans, one can conceptualize 'chemical insults' to the brain and make some inferences about a possible neurochemical basis of delirium despite the probability that these different drug manipulations may represent distinct neurochemical routes to delirium.

ACh receptor antagonists trigger generalized EEG slowing (most prominently increased delta frequencies $(1-3 \mathrm{~Hz})$ and decreased alpha frequencies $(8-12 \mathrm{~Hz}))$ in humans, which is associated with cognitive and attentional changes ${ }^{148}$ and, in some studies, with frank delirium $^{80}$. Similarly, the cholinergic antagonists atropine and scopolamine slow the average cortical EEG frequency to about $8 \mathrm{~Hz}$ while also impairing performance in a rewarded maze task ${ }^{149}$. Anticholinergic effects are ascribed to many common medications and there is evidence that increased anticholinergic burden increases delirium risk ${ }^{150,151}$. These data indicate that robust disruption of cholinergic function can be sufficient to trigger delirium but does not imply that cholinergic impairment occurs in all deliria. To date, acetylcholinesterase (AChE) inhibitors have not been demonstrated to reduce the occurrence, severity or duration of delirium in adult orthopaedic fracture or surgery patients or in critically ill patients ${ }^{152-154}$. Evidence exists for increased mortality with rivastigmine (a cholinesterase inhibitor) treatment in critically ill patients ${ }^{154}$ but these patients likely do not have a pre-existing cholinergic deficit. Thus, there is no clear biological basis for preventing delirium in ICU patients by correcting the levels of a single neurotransmitter. Conversely, acute systemic inflammation more readily triggers cognitive dysfunction in mice with prior lesions of the basal forebrain cholinergic system, and AChE inhibitors are protective in these mice ${ }^{155}$ as well as in patients with Lewy body dementia who have established cholinergic degeneration and delirium-like symptoms ${ }^{156}$. Therefore, acutely impaired cholinergic function can trigger delirium and might strongly contribute to delirium symptoms but this effect is most likely to emerge in patients (or animals) with existing cholinergic vulnerability. However, leveraging this observation for treatment of delirium through the identification of patients with cholinergic deficiency is more complicated. Studies of cholinergic system integrity or disruption during delirium have not been performed, but PET and SPECT imaging methods for vesicular ACh transporter, AChE and cholinergic receptor subtypes are becoming available ${ }^{157}$ and may offer routes to stratifying patients by cholinergic system integrity.

Anti-psychotic drugs, which largely function by blocking D2 dopamine receptors, have long been used in delirium treatment. However, as prevention and treatment trials have failed to show benefit with various antipsychotic agents, including haloperidol, olanzapine or ziprasidone, in preventing or treating delirium in multiple settings ${ }^{158-161}$, a hyper-dopaminergic theory of delirium is significantly undermined. In general, the hyperdopaminergic state is characterized by locomotor hyperactivity, as demonstrated in experimental animals ${ }^{162}$, and many clinicians continue to use antipsychotic drugs to reduce agitation and/or psychological distress (for example, hallucinations or delusional thoughts) in patients with delirium; however, there remains little evidence to support this practice. Earlier studies producing experimental delirium in humans by cholinergic inhibition suggest that the dopamine receptor D1 and D2 antagonist chlorpromazine produced a marked psychomotor inhibition but further potentiated EEG slowing ${ }^{80}$. Thus, dopaminergic status may affect the psychomotor state during delirium but is unlikely to have a mechanistic role in delirium at the syndromic level.

Patients who fulfil the criteria for a delirium diagnosis can be profoundly hypoactive or agitated and hyperactive. Therefore, delirium encompasses very different states of arousal and it is tempting to implicate changes in the brain's major arousal systems in the delirium syndrome. Histamine influences arousal by activating hypothalamic tuberomammillary nucleus projections to the prefrontal cortex (PFC), limbic system and basal ganglia, and multiple studies show the importance of histamine in arousal and wakefulness ${ }^{163}$. Although preclinical studies have not specifically addressed delirium, first-generation antihistamines (H1 receptor antagonists) have well-described sedative effects, lowering brain arousal state, and delirium is a documented adverse effect of both $\mathrm{H} 1$ and $\mathrm{H} 2$ receptor antagonists ${ }^{164}$.

Noradrenaline has profound effects on PFC activity. Noradrenergic neurons in the locus coeruleus are silent during rapid eye movement sleep and show prominent phasic firing during alert wakefulness ${ }^{165}$. Conversely, during stress, activation of the amygdala activates the locus coeruleus to trigger high tonic noradrenergic activity, driving poor attentional performance ${ }^{165}$. In this situation, cognitive and behavioural function switches from thoughtful 'top-down' regulation by the PFC to more reflexive, emotional responses (such as fear and threat) driven by the amygdala. Therefore, both too much and too little noradrenergic activity impairs frontal cortical function ${ }^{166}$ and it is tempting to speculate that these divergent states of arousal may respectively contribute to hyperactive and hypoactive states in delirium. Degeneration of the locus coeruleus occurs early in Alzheimer disease and impaired forebrain noradrenergic tone correlates with increased aggression and impaired cognition ${ }^{167}$. Acutely, activation of the sympathetic nervous system, which results in elevated noradrenergic activity, occurs in inflammatory trauma and sepsis and in psychological stress ${ }^{168,169}$, and elevated blood 
noradrenaline was significantly associated with postoperative delirium in elderly patients undergoing major surgery ${ }^{170}$. Of note, the hypothalamic-pituitary axis is usually activated in tandem with the sympathetic nervous system and increased cortisol has been associated with delirium in a number of settings. Interestingly, cognitive deficits associated with thoracic trauma in mice are blocked with a corticotropin-releasing hormone receptor 1 (CRHR1) antagonist, which prevents cortisol release ${ }^{171}$.

Excessive noradrenergic drive is also present during alcohol withdrawal ${ }^{172,173}$ and accounts for several of its symptoms, such as high blood pressure, tremor and agitation. Excessive noradrenergic drive can be treated using noradrenaline release-limiting $\mathrm{a}_{2}$-adrenergic receptor agonists (such as clonidine and dexmedetomidine ${ }^{174}$ ), which have been proposed for the prevention of delirium during alcohol withdrawal ${ }^{175}$ as well as in ICU patients and after cardiac surgery in adult patients or non-cardiac surgery in elderly patients ${ }^{176-178}$. Furthermore, opioids, which pose a risk for delirium ${ }^{52}$, also increase noradrenaline release in the $\mathrm{PFC}^{179}$. Considerable research is required in animals and in humans to clarify the roles of noradrenaline and stress hormones in delirium.

Despite their utility in treating alcohol withdrawal, benzodiazepines are also implicated in delirium. It is abundantly clear that benzodiazepines produce acute cognitive impairment and that increased GABAergic tone in the corticothalamic network is a major driver of unconscious brain states and is the basis of a hypothetical model of delirium pathogenesis ${ }^{180}$. Although once regarded as an appropriate treatment for delirium, benzodiazepines increase the risk for transition to delirium in ICU patients ${ }^{181,182}$. Several studies now suggest that dexmedetomidine is less deliriogenic than GABAergic sedatives in ICU and surgical patients but this evidence has not yet reached consensus ${ }^{183,184}$.

Neuroanatomical substrates and failure of network connectivity. Neurochemical influences on brain state are dependent on the neuroanatomical networks in which these neurotransmitters operate. Structural connectivity studies show that loss of integrity in the inter-hemispheric corpus callosum is associated with increased delirium duration ${ }^{185}$, and diffusion tensor imaging demonstrated that abnormalities in the hippocampus, thalamus, basal forebrain and cerebellum (and associated white matter tracts: fimbria, fornix, internal capsule, corpus callosum) are correlated with delirium incidence and severity ${ }^{186}$. These results are consistent with data from animal studies showing that prior synaptic and axonal damage in the hippocampus, thalamus and cholinergic basal forebrain all increase the risk of acute cognitive deficits after acute systemic inflammation ${ }^{45,155,187}$ and human CSF studies suggest that further synaptic damage seems to occur in acute infection ${ }^{188}$.

Regardless of primary aetiology, it is hypothesized that impaired neuronal network connectivity may be the final driver of the delirium syndrome; that is, underlying predispositions and superimposed stressors might combine to cause a failure of functional connectivity in neural networks, leading to a profound failure of normal brain function ${ }^{189}$. Brain networks can be studied by analysing statistical relationships between time series of neural activity recorded from different brain areas using functional MRI (fMRI) or EEG ${ }^{190}$. In normal conditions, activity in the dorsolateral prefrontal cortex (part of the executive network) is negatively correlated with activity in the posterior cingulate cortex ${ }^{191}$, which is part of the default mode network that is selectively active at rest and during internally directed thought and becomes deactivated during externally directed tasks ${ }^{192}$. During delirium, a reversal of the relationship between these two reciprocal networks was observed, perhaps contributing to clinical features such as inadequate shifting and focusing of attention. In addition, reduced functional connectivity of subcortical regions was observed during delirium, which might be related to reduced arousal ${ }^{190}$. In addition to this approach, in which fMRI is used to study the level of functional connectivity between a selected seed region of interest and any other brain region, brain networks can be studied globally, using graph theory and $\mathrm{fMRI}$ or EEG. During delirium, a variety of network changes have been observed, such as reduced connectivity strength, reduced global efficiency, reduced local clustering and reduced modularity ${ }^{193-196}$.

In conclusion, delirium is associated with weakened brain networks that become less integrated. Available tools must now be applied to characterize impaired network connectivity during delirium in different patient groups to examine convergence, synergy or divergence between degenerative, metabolic, inflammatory and pharmacological pathways to delirium.

\section{Diagnosis, screening and prevention}

The term delirium appeared in the DSM-III (published in 1990 $)^{27}$ and has been present with shifting criteria in subsequent DSM editions. With the advent of DSM-IV (published in 1994) ${ }^{197}$, delirium criteria were based on the novel initiative of tallying the frequency of clinical symptoms in patients deemed to be delirious by psychiatrists. As such, DSM-IV was the first attempt to create a scientifically based psychiatric evaluation for delirium, as diagnostic clinical elements served as the basis for deciding which criteria should be included in the diagnosis construct. In the current edition of the DSM, DSM-5 (REF. ${ }^{4}$ ), among five criteria (A-E), the presence of disturbances in attention and awareness (criterion A; for example, reduced orientation to the environment or altered arousal ${ }^{198}$ ) and at least one other cognitive deficit (criterion C) that has developed over a short period, specified as "usually hours or days" (criterion B), are required for a delirium diagnosis. Coma is excluded as a disturbance of attention or awareness but the guidance notes state that patients above the level of coma who are unable to produce speech or engage in cognitive testing or interview should be classified as having 'severe inattention' and thus fulfil criterion A. Criteria D and E relate to the exclusion of alternative explanations for the disturbances in criteria $\mathrm{A}$ and $\mathrm{C}$, such as other neurocognitive disorders (criterion D) or medical conditions, drug use or withdrawal, or toxin exposure (criterion E). 
The International Classification of Disease 10th Edition (ICD-10; published in 1993) criteria for delirium require the presence of symptoms from several domains: consciousness and attention, global disturbance of cognition, psychomotor disturbance, sleep-wake cycle disruption and emotional disturbances. In research and clinical practice, the DSM criteria have been more commonly employed, possibly because the ICD-10 criteria are more restrictive ${ }^{199}$. For example, a study of 230 older medical inpatients found that $24.9 \%$ fulfilled the DSM-IV criteria whereas $10.1 \%$ fulfilled the ICD-10 criteria for delirium ${ }^{200}$.

The diagnostic process involves two fundamental steps. First, a bedside clinical assessment of the patient is performed to ascertain the level of attention and arousal and the presence of other cognitive deficits, psychotic features or other mental status abnormalities. Second, evidence of acute change from baseline attention and awareness, which may fluctuate in presence and severity, is sought from the patient, caregivers or staff who know the patient, from medical records or, less commonly, from the clinician's own knowledge of the patient. The clinician may also deduce that there is an acute change in some circumstances, for example, if there is significant acute drowsiness in a patient who is normally functionally independent. After diagnosis, further assessment of the patient to obtain more detail about the individual profile of delirium, including features such as delusions, hallucinations or mood changes, is crucial because this information influences the specific management plan.

\section{Assessment tools}

In clinical practice and in some research studies, the diagnosis and assessment process is based on DSM-5 without the use of additional tools, although tools are commonly used to formalize and help standardize assessment. More than 50 delirium assessment tools have been developed ${ }^{201-205}$, with various intended purposes, including for episodic use at first presentation or at other times when delirium is suspected, to monitor regularly for new-onset delirium in inpatients (usually carried out daily or more often), for 'ultra-brief' screening, for detailed phenomenological and/or neuropsychological assessment, and to measure delirium severity. The diagnostic performance of most tools compared with reference standard assessment has been reported, although these findings are open to interpretation owing to the considerable variability of reference standards ${ }^{206}$ used and the exclusion in some studies of patients who are unable to communicate verbally, meaning that the sample is unrepresentative of the whole spectrum of delirium. Here, we provide a summary of some of the tools that are commonly used in research and clinical practice. DSM-5-based criteria and common delirium screening tools are summarized in FIG. 5.

Assessment of episodic delirium in non-ICU settings. The Confusion Assessment Method (CAM; The Hospital Elder Life Program (HELP)) is based on four features from the 1987 DSM-III-revised criteria ${ }^{207}$ : acute onset and fluctuating course (criterion A), inattention (criterion $\mathrm{B})$, disorganized thinking (criterion $\mathrm{C}$ ) and level of consciousness (criterion D). Trained raters perform a cognitive test and then an interview and score each feature as present or absent, with the entire process taking 5-10 minutes. A positive CAM score requires the presence of criteria A and B and either or both of criteria $\mathrm{C}$ and $\mathrm{D}$. The diagnostic performance of CAM has been assessed in 23 studies (2,629 patients in total), with sensitivities that varied based on the study setting (from 0.09 to 1.0 ) and the training of the raters, and with moderate-to-high specificities of 0.84 to 1.0 $\left(\right.$ REFS $\left.^{208,209}\right)$. The CAM is the most-used tool in research studies ${ }^{210}$ and is also commonly used in clinical practice, although, in this setting, its sensitivity is often lower, particularly when used without the recommended prealgorithm cognitive test and interview ${ }^{209,211-213}$. The brief CAM (bCAM) is a 2-minute, 4-item variant of the CAM designed and validated for use in the emergency department. Another variant of the CAM, 3D-CAM, is a 2-5 minute (median of 3 minutes), 20 -item tool comprising a cognitive test, interview and observation questions. Both bCAM and 3D-CAM have generally good psychometric performance ${ }^{214,215}$.

The 4 A's test (4AT) is a 2 minute, 4 -item tool designed for use in clinical practice and that does not require special training to administer ${ }^{216}$. The four items are alertness, cognition (a short test of orientation), attention (recitation of the months in backwards order), and the presence of acute change or fluctuating course. A meta-analysis of 17 diagnostic test accuracy studies ( $n=3,701$ observations) reported a pooled sensitivity and specificity of $88 \%{ }^{217}$. In a randomized controlled trial, the 4AT had higher sensitivity than and similar specificity to the $\mathrm{CAM}^{208}$. The $4 \mathrm{AT}$ is mandated for all hip fracture patients in England and whole clinical population data from 2018 (total $n=60,000$ patients) showed that $95 \%$ of patients were screened with the $4 \mathrm{AT}$, with $25 \%$ having a positive score ${ }^{218}$.

The 12-item Stanford Proxy Test for Delirium (S-PTD $)^{219}$ is a screening tool intended to be performed by nurses in a non-ICU population. The nurse assesses the patient for the presence of items suggestive of delirium in the previous 8 hours ${ }^{219}$, with 3 or more items suggesting the presence of delirium ${ }^{219}$. The S-PTD has been validated against neuropsychiatric assessment by a psychiatrist and has a sensitivity of 0.8 and a specificity of 0.9 (REF. ${ }^{219}$ ).

Monitoring for new-onset delirium in inpatients. Tools to monitor for new-onset delirium in inpatients are employed regularly and frequently, once or more daily, on an ongoing basis. Tools assessing the level of arousal include the Richmond Agitation Screening Scale (RASS $^{220}$ and the modified RASS ${ }^{221}$. Some tools record the level of arousal and acute cognitive change, for example, the National Early Warning Score 2 (NEWS2), which is used throughout the National Health Service in the UK ${ }^{22}$. The Royal College of Physicians in the UK have recommended that the Single Question in Delirium (one variant is: "Is the person more confused or more drowsy than usual?") is used to prompt completion of the NEWS2 chart ${ }^{222}$. The Recognizing Acute Delirium As part of your Routine (RADAR) 
Symptoms suggestive of delirium (or proactive assessment in high-risk patients) Acute disturbance of attention, reduced environmental awareness, altered arousal and/or change in cognition

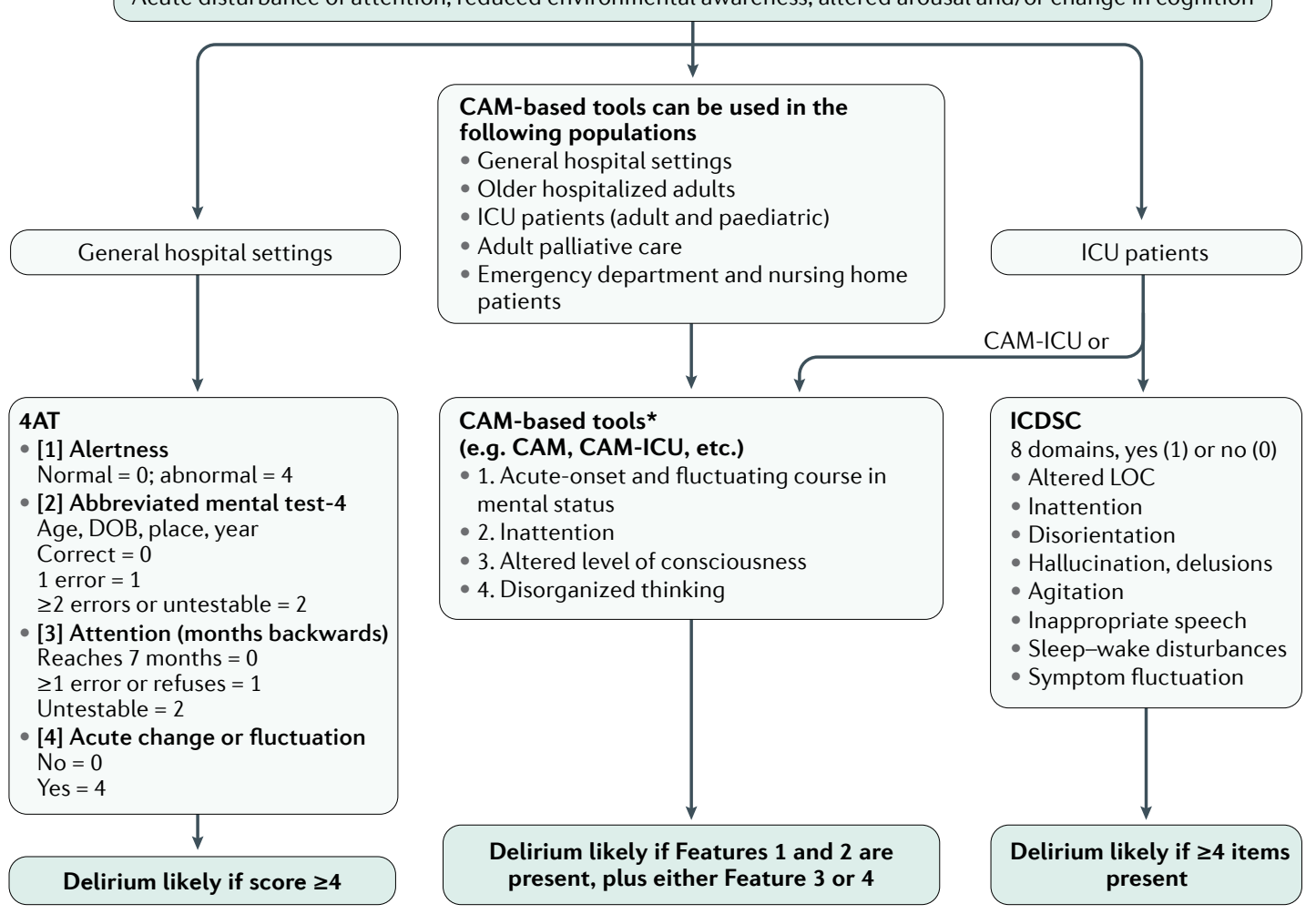

Fig. 5 | Common tools to screen for delirium in different settings. Once there is suspicion of delirium based on the presence of symptoms or when proactive screening is indicated, such as in high-risk clinical situations, the choice of screening tool is made based on the setting. The 4A's test (4AT) is a 4-item test used in general hospital settings; a score $\geq 4$ indicates delirium is likely. Confusion Assessment Method (CAM)-based tools, such as the CAM, CAM-Intensive Care Unit (ICU), brief-CAM, paediatric-CAM and preschool-CAM, assess four features, Features 1-4 (or Features A-D depending on the specific tool). For delirium to be present according to CAM-based tools, Features 1 and 2 must be present, plus either Feature 3 or 4 . The Intensive Care Delirium Screening Checklist (ICDSC) is a delirium assessment tool measuring 8 domains, recorded as yes (present; score 1 ) or no (absent; score 0 ) answers. Delirium is likely for a score of $\geq 4$. The clinical tools here use varying combinations of bedside testing and information on change in mental status in the period preceding the assessment. *Depending on the tool used, Features 1-4 may be called A-D and will be assessed in different ways. DOB, date of birth; LOC, level of consciousness.

tool prompts nurses to look for any change in cognitive function and level of arousal at the point of drug administration on the $\operatorname{ward}^{223}$. There are also several longer monitoring tools that are designed to be completed by nurses at the end of each shift, including the Delirium Observation Screening Scale (DOS) ${ }^{224}$ and the Nursing Delirium Screening Scale (Nu-DESC) ${ }^{225}$. For most monitoring tools, either the sensitivity or the specificity is limited, so it is recommended that a positive score with any of these tools should be followed up with a more definitive episodic tool or with diagnostic assessment.

Delirium assessment in the ICU. In a systematic review $^{226}$ that included 36 studies of ICU patients and 5 different delirium assessment tools, the CAM for the ICU (CAM-ICU) and the Intensive Care Delirium Screening Checklist (ICDSC) were shown to be the most valid and reliable tools for delirium assessment in critically ill adults. The CAM-ICU is specifically designed to be used in critically ill patients, including those on mechanical ventilation ${ }^{227}$, when delirium is suspected; however, the tool developers recommend that assessment is performed once or more daily. CAM-ICU comprises the four CAM items and a scoring algorithm, with the difference that attention and disorganized thinking are assessed using embedded short cognitive tests and yes/no interview questions. In a meta-analysis of 9 studies including 969 patients, the CAM-ICU had a pooled sensitivity of $80 \%$ and a pooled specificity of $96 \%$.228.

The ICDSC ${ }^{229}$ comprises eight features: level of consciousness, inattention, disorientation, psychosis, psychomotor changes, speech or mood changes, sleepwake cycle disturbance, and symptom fluctuation. Each item is rated 0 (absent) or 1 (present) at the end of each nursing shift, with scores of $\geq 4$ considered to indicate delirium. In a meta-analysis of 4 studies including 361 patients, the ICDSC had a pooled sensitivity of $74 \%$ and a specificity of $82 \%{ }^{228}$.

Both of these tools were recommended by the 2013 Pain, Agitation and Delirium Clinical Practice (PAD 178) Guidelines and the 2018 Clinical Practice Guidelines for the Prevention and Management of Pain, Agitation/ Sedation, Delirium, Immobility, and Sleep Disruption 
in Adult Patients in the ICU (PADIS 179) for use in critically ill, ventilated patients.

Ultra-brief screening tools. These tools are designed to be used to quickly assess the patient at the bedside, typically taking less than a minute to administer. They can be used for a brief assessment, followed by a more detailed assessment if positive. For example, the Delirium Triage Screen assesses a patient's ability to spell the word 'lunch' backwards and also notes if they have an altered level of arousal; it has good sensitivity and moderate specificity ${ }^{215}$. The Ultra Brief 2 Item Screener (UB-2) was designed as a $<1$ minute, 2 -item delirium screen consisting of 2 questions: "Please tell me the day of the week?" and "Please tell me the months of the year backwards starting at December?" If either question is answered incorrectly, delirium is suspected and a more definitive episodic tool is performed ${ }^{230}$. The Simple Question for Easy Evaluation of Consciousness (SQEEC) tool involves asking the patient to name a place they would like to visit that they have not visited before and then to describe how they would make the journey. In the initial evaluation study, this question showed both good sensitivity and specificity ${ }^{231}$. Tools based on cognitive testing are not appropriate for regular monitoring because of practice effects and patient burden.

Detailed phenomenological or neuropsychological assessment. The Delirium Rating Scale-revised-98 (DRS-R98) tool ${ }^{232}$ is a detailed phenomenological assessment tool that consists of 16 items ( 13 for severity and 3 for diagnosis) and takes 20-30 minutes to perform. Multiple domains are covered, including sleep-wake cycle disturbance, hallucinations, lability of affect, attention and visuospatial ability. The DRS-R98 yields a numerical score relating to both the presence and severity of rated items and provides a good balance of sensitivity and specificity, with high reliability, especially when used in longitudinal investigations ${ }^{233}$. The DRSR98 is often used as part of a formal reference standard assessment, with its features being used to inform DSM-IV or DSM-5 criteria. The Cognitive Test for Delirium ${ }^{234}$ comprises a 5-domain battery of tests covering orientation, attention, memory, comprehension and vigilance. This test has been validated in ICU patients, showing high sensitivity and specificity. The DelApp is a computerized, objective test of arousal and attention that is implemented on a smartphone and is for use in both the general population and ICU patients. Case-control studies show a high sensitivity and a moderate-to-high specificity of DelApp in the detection of delirium ${ }^{235,236}$.

Assessing delirium severity. Delirium severity is a complex concept relating to the scale of several potential parameters, such as the extent of cognitive impairment, the level of arousal, the duration of delirium, the number of delirium criteria present and the level of distress experienced by patients ${ }^{237}$. The three main instruments that are most frequently used are CAM-based instruments ${ }^{207}$, the DRS-R98 (REF ${ }^{232}$ ) and the Memorial Delirium Assessment Scale (MDAS) ${ }^{238}$. The CAMSeverity (CAM-S) score ${ }^{239}$ was designed to quantify the intensity of delirium symptoms on a scale of 0-7 in the short form and of $0-19$ in the long form. The MDAS is a 10-item, 4-point (0-3) rated scale with a range of 0 to $30\left(\right.$ REF. $\left.^{238}\right)$. The MDAS and the DRS-R98 (REF $\left.{ }^{237}\right)$ are used for delirium assessment as well as to measure delirium severity. Although delirium severity, as measured by these delirium severity tools, is thought to be associated with worse outcomes, such as mortality or long-term cognitive impairment, this is not known. Apart from duration of delirium as an index of severity ${ }^{3,240}$, no other severity measures have been correlated with mortality or cognitive decline.

\section{Clinical investigations of delirium}

The initial assessment of delirium involves a detailed general review that considers multiple possible triggers of the delirium syndrome. Therefore, investigation initially involves various standard clinical tests, such as routine blood tests and radiological tests, that are guided to some extent by the presenting features ascertained from the patient's medical history and physical examination. Importantly, some delirium presentations are caused by primary central nervous system disorders and, in a minority of cases in which the features suggest this possibility, various brain-specific tests, including CT and MRI scans, EEG, lumbar puncture and antibody tests for autoimmune encephalitis, are indicated ${ }^{241}$. CT scanning for all delirium cases is not justifiable; for example, in a study of 1,653 patients with delirium (median age 80 years, interquartile range $71-86,54 \%$ male) admitted to hospital within an 18-month period, among those patients receiving CT scans, only $11 \%$ had positive CT findings, with haemorrhage being the most common aetiology contributing to delirium ${ }^{242}$.

Research studies indicate the possibility of future expansion of brain-specific investigations for delirium diagnosis in clinical practice. Functional neuroimaging utilizing CT and MRI perfusion scans revealed reduced cerebral blood flow, cerebral oxygenation and abnormalities of glucose uptake that could be related to delirium $^{243}$. An updated systematic review suggested a consistent association of delirium with white matter hyperintensity ${ }^{243}$. More recently, a unique pattern in cerebral glucose hypometabolism was observed on FDG-PET scans of hospital patients with delirium ${ }^{46}$. These changes resolved with the resolution of delirium whereas they persisted in patients with dementia ${ }^{244}$.

Recommendations from guidelines currently suggest that EEG should be conducted if there is suspicion of non-convulsive status epilepticus and that lumbar puncture should be performed where central nervous system infection or autoimmune disorders are suspected ${ }^{241}$. In one study, EEG abnormalities in frail, elderly hospitalized adults with delirium revealed occipital slowing, peak power and alpha decrease, delta and theta power increase, and slow wave ratio increase during active delirious states ${ }^{245}$. Furthermore, EEG measures correlated significantly with cognitive performance and delirium severity ${ }^{245}$.

Neurophysiological correlates of delirium have been studied using EEG and quantitative EEG in small cohorts of patients ${ }^{246,247}$. In a cohort of non-sedated cardiothoracic 
surgery patients, eyes-closed EEG recording with two electrodes in a frontal-parietal derivation could distinguish delirious from non-delirious patients ${ }^{246}$. In a retrospective case-control study, quantitative EEG could distinguish delirious from non-delirious patients with a sensitivity of $100 \%$ and a specificity of $99 \%{ }^{247}$. While advances in neuro-functional imaging and neurophysiology are likely to expand our understanding of delirium pathophysiology and the mechanistic basis of possible future treatments, the role of EEG in delirium screening and diagnosis remains undefined.

\section{Challenges in detecting delirium}

Delirium remains grossly under-diagnosed and undetected ${ }^{248}$, with fewer than half of delirium cases in hospital typically being detected ${ }^{248}$. Multiple reasons exist for under-diagnosis, including, amongst others, a general lack of delirium training at all levels including in undergraduate education ${ }^{249}$, attitudes such as the perception that delirium is not 'owned' by (that is, the responsibility of) certain specialties or groups of practitioners ${ }^{250}$, the use of imprecise alternative terms such as 'confusion', and a lack of perception that delirium is important. It is now clear that successful implementation of delirium detection, treatment and risk reduction is a complex challenge ${ }^{251}$, requiring an educational programme addressing both attitudes and skills, supported by audit, and using tools with proven implementability and tailored to the population to be tested. A study using quality improvement methodology to develop a digital pathway demonstrated the promise of this combined approach to improve delirium assessment and detection ${ }^{252}$.

\section{Box 2 | Delirium prevention in different health-care settings}

Consensus guidelines ${ }^{241,262}$ make a number of recommendations for delirium prevention in various health-care settings.

\section{General settings}

Multicomponent interventions

- Early recognition of high-risk factors (age $>65$ years, dementia, hip surgery and high acuity)

- Daily screening for delirium

- Environmental orientation (sensory, auditory, dentures, time, events, family visits and music)

- Maintain normal hydration

- Regulation of bladder and bowel function

- Early establishment of normal diet

- Correction of metabolic disorders

- Cardiorespiratory optimization (with provision of oxygen if appropriate)

- Early identification of infection

- Effective treatment of pain

- Daily mobilization

- Avoidance of antipsychotic drugs

- Avoidance of benzodiazepines

- Reduced nocturnal disturbances to promote sleep
- Early removal of devices (intravascular and airway devices)

- Avoidance of physical restraints

- Sleep promotion (eye mask and earplugs)

Pharmacological interventions

- None with high-level evidence

Intensive care and high acuity units, intubated and non-intubated patients

Non-pharmacological interventions (as above, consider also)

- Early recognition of high-risk patients (age $>65$ years, high acuity, sepsis, shock, dementia and ventilation)

- Light sedation

- No benzodiazepines

- Early mobilization

- Promotion of day-night routine

- Environmental awareness and orientation

- Removal of devices (intravascular and airway devices)

Pharmacological interventions

- Some suggestive evidence but not recommended by consensus guidelines
In approaching the challenges involved in achieving satisfactory rates of delirium detection, it is helpful to consider different settings and the different stages in the patient journey. Delirium is a common medical emergency, affecting $25 \%$ of older medical patients ${ }^{16}$. Delirium is also common after elective surgery in older patients. Therefore, it is reasonable to proactively assess for delirium in these populations and settings at key points in the journey, including on admission and after surgery using a short episodic tool, such as the 4AT or bCAM. Delirium also commonly arises in medical inpatients after admission but it is not feasible to use episodic tests regularly (one or more times daily) for extended periods in these patients because of patient burden and cognitive test practice effects. The use of mostly observational monitoring tools, such as NEWS2 or the Delirium Observation Scale, is more suitable, with episodic tools or a clinical assessment being used if delirium is detected with the monitoring tool. A combination of NEWS2 for monitoring and the 4AT for more detailed assessment is recommended across the UK National Health Service in high-risk patients ${ }^{222}$. Another model that has been implemented in hip fracture care is to use the Single Question in Delirium (SQiD) tool followed by the $4 \mathrm{AT}$ if positive ${ }^{253}$. ICU patients present particular challenges and bespoke tools, such as the CAM-ICU and the ICDSC, are recommended. The Network for Investigation of Delirium: Unifying Scientists (NIDUS) is an excellent resource for delirium screening and severity tools. ICU delirium-specific tools and resources can be found at the Critical Illness, Brain Dysfunction and Survivorship (CIBS) Center.

\section{Prevention}

Non-pharmacological interventions. There is good evidence that multicomponent interventions can reduce the risk of incident delirium in at-risk hospital inpatients and such interventions are recommended in the Scottish Intercollegiate Guidelines Network (SIGN) guidelines ${ }^{241}$. Intervention packages vary across studies but include components such as physiotherapy, reorientation, cognitive stimulation, early mobilization, non-pharmacological promotion of sleep, correction of sensory impairments, identification and treatment of underlying causes or postoperative complications, pain management, avoidance of constipation, hydration, nutrition, and oxygen delivery (BOX 2).

The Hospital Elder Life Program (HELP) is a multicomponent delirium prevention protocol that systematically addresses visual and hearing impairment, immobility, disorientation, sleep deprivation (non-pharmacological) and dehydration. The intervention is delivered by a team that includes trained nurses, physicians and volunteers. In a prospective matched-pair (non-randomized controlled trial) study involving 852 general medical patients $\geq 70$ years of age ${ }^{254}$, the HELP protocol resulted in a $5 \%$ absolute reduction in the risk of developing delirium. A 2018 meta-analysis of 14 HELP studies broadly confirmed these findings and found a reduced rate of falls ${ }^{255}$. In 2013, HELP was expanded by the addition of protocols addressing hypoxia, infection, pain and constipation because these aspects were 
included in the 2010 UK National Institute for Health and Care Excellence (NICE) guidelines on delirium prevention, diagnosis and management ${ }^{256}$. Another study involving 126 postoperative hip surgery patients ${ }^{257}$ found that a proactive geriatrics consultation, which was designed to identify and minimize delirium risk factors, such as exposure to deliriogenic medications, urinary catheters, immobility and inadequate nutrition, reduced the incidence of delirium by $18 \%$. A 2020 meta-analysis of 8 randomized controlled trials involving 2,105 patients in medical, surgical and ICU settings found that delirium prevention protocols reduced overall delirium risk (risk ratio $0.53 ; 95 \%$ CI $0.41-0.69)^{258}$, with no clear evidence of an effect on duration of delirium, length of stay, falls or mortality; 3 of the 8 studies were based on the HELP protocol. In a planned comparison, there was no difference between HELP-based and other protocols, suggesting that protocols that do not require volunteers can be as effective as those using volunteers. In support of this conclusion, a study showed that fidelity to a comprehensive delirium prevention protocol in routine practice could be achieved without volunteers ${ }^{259}$. A Cochrane review examined the evidence for delirium prevention in institutional long-term care ${ }^{260}$; three cluster-randomized, controlled trials were considered, none of which included pharmacological intervention, and only one study identified pharmacist-led medication review as an intervention that probably prevented delirium.

The ICU is a particularly deliriogenic environment, with patients being exposed to more than ten delirium risk factors (on average) during their critical illness ${ }^{261}$, many of which are amenable to modification. Although many baseline risk factors, such as advanced age, are not modifiable, others can be addressed ${ }^{18}$. Vision and hearing impairment ${ }^{27}$, for example, can be improved with eyeglasses and hearing aids and the risk of delirium due to baseline cognitive impairment may be mitigated by frequent reorientation. In addition, many acute risk factors can be avoided or reduced. Immobility can be avoided by minimizing the use of physical restraints and employing early mobility protocols; hypovolaemia, electrolyte abnormalities and infection are amenable to acute interventions; and the risk attributable to deliriogenic sedatives, such as benzodiazepines, can be avoided by minimizing sedation in general and by using alternative medications ${ }^{262}$.

Studies in the critical care setting have shown that early physical and occupational therapy during daily breaks in sedation in mechanically ventilated medical ICU patients, in comparison to the control group of daily interruption of sedation with therapy as ordered by the primary care team (usual care), led to a shorter delirium duration in the ICU (median 2.0 days, interquartile range $0.0-6.0$ for the intervention group versus 4.0 days, $2.0-8.0$ for the control group; $P=0.02)^{263}$. Similarly, early mobilization of surgical patients in the ICU led to improved functional status and fewer days spent in the ICU with delirium compared with the control group (standard of care) $(P=0.0161)^{264}$. These preventive strategies have been bundled into a process of care, such as the ABCDEF bundle, that promotes awake and spontaneously breathing, mobile patients ${ }^{265}$. Compliance with the ABCDEF bundle is associated with a reduced risk of delirium on the following day (adjusted OR 0.60; 95\% CI 0.49-0.72) and multiple other important outcomes in critically ill patients ${ }^{266}$ (FIG. 6). Further work quantifying the effectiveness and magnitude of possible benefits of such bundles needs to be confirmed in proper randomized prospective studies ${ }^{267}$.

Pharmacological interventions. Multiple studies have evaluated the possibility that antipsychotic drugs may be effective in preventing delirium in high-risk patients. A 2019 systematic review examined 14 randomized controlled trials or prospective observational studies comparing antipsychotic drugs with placebo or other antipsychotic drugs (for example, first-generation antipsychotic drugs compared with second-generation or atypical antipsychotic drugs $)^{268}$, concluding that the evidence does not support the use of haloperidol or second-generation antipsychotic drugs for the prevention of delirium ${ }^{268}$. Studies evaluating melatonin have been mostly small, with considerable methodological heterogeneity, and have shown mixed effects on delirium prevention ${ }^{269}$. A Cochrane review published in 2016 found no clear evidence that cholinesterase inhibitors, antipsychotic medication or melatonin were effective in delirium prevention in non-ICU patients ${ }^{270}$.

\section{Management}

With delirium affecting at least one in seven hospitalized patients overall and around half of ICU patients, delirium treatment is a major part of the workload of a hospital. Delirium treatment is complex, as it involves addressing multiple domains ${ }^{241,262}$. This multidomain approach can be summarized as follows: addressing the often multiple delirium triggers ${ }^{81}$, correcting physiological disturbances, treating the symptoms of delirium including distress, communicating with patient and carers, and addressing the current and future risks linked with delirium. Addressing each of these domains requires highly skilled systematic care from a multidisciplinary team.

Delirium treatment is traditionally presented as two different approaches: 'non-pharmacological' and 'pharmacological' approaches. However, this is a flawed idea, as the non-pharmacological approach is in fact a multidomain approach that can include the use of drugs to treat some aspects of the delirium syndrome, such as severe agitation. The pharmacological approach is conceptually drawn from drug-based treatment approaches in other conditions, such as depression and bipolar illness, and is based on prescribing a drug or combination of drugs to treat delirium as a syndrome rather than on treating individual symptoms. The pharmacological approach is not supported in current guidelines or by the evidence; that is, there is no consensus that diagnosed delirium should be treated with one or more drugs. Rather, the pharmacological approach is mostly a concept that has been applied in clinical trials. In practice, multidomain treatment (which can infrequently involve drug treatment) is the basis of all delirium treatment and its use in different settings is discussed below. 
a Mechanical ventilation

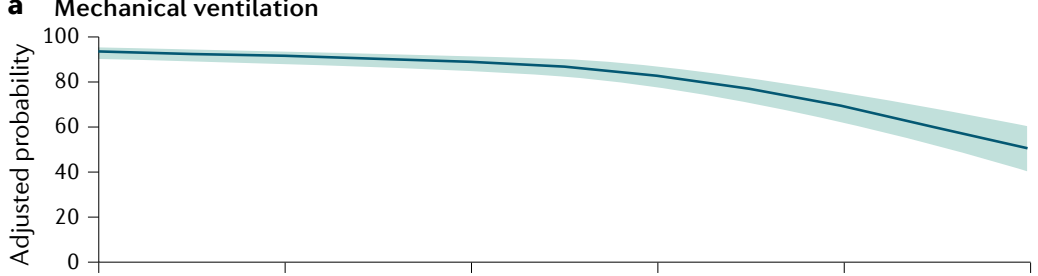

b Significant pain

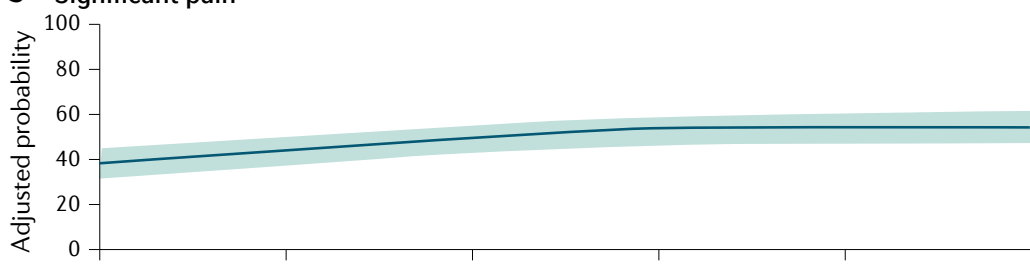

C Coma

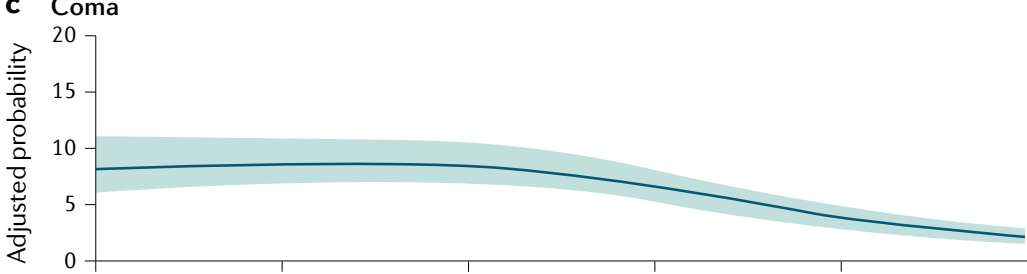

d Delirium

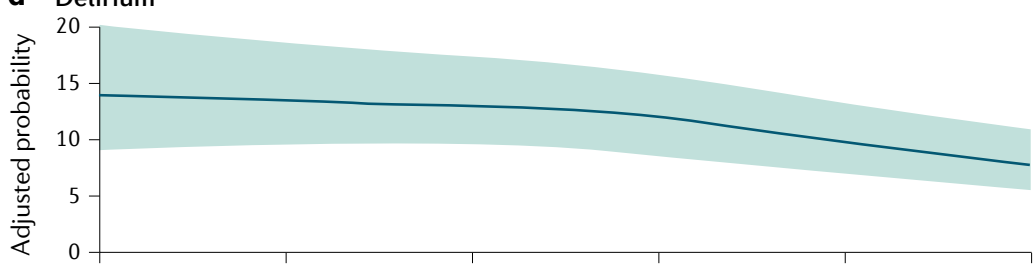

e Physical restraints

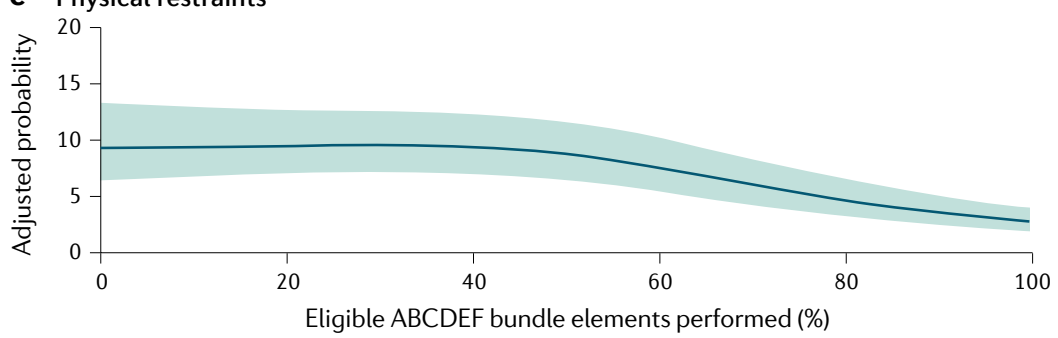

Eligible ABCDEF bundle elements performed (\%)

Fig. 6 Associations between performance of the ABCDEF bundle and outcomes. Results from the Intensive Care Unit (ICU) Liberation Collaborative of over 15,000 patients from 68 academic, community and federal ICUs, which showed the percent performance of the $A B C D E F$ bundle and symptom-related outcomes ${ }^{266}$. ABCDEF bundle components include $A$ (Assess, prevent and manage pain), $B$ (Both spontaneous awakening and breathing trials), C (Choice of analgesia and sedation), D (Delirium: assess, prevent and manage), $\mathrm{E}$ (Early mobility and exercise) and $\mathrm{F}$ (Family engagement and empowerment). Each graph shows the relationship between the proportion of eligible elements of the ABCDEF bundle performed on a particular day and the probability that the patient would experience that symptom-related outcome on the following day. The outcomes include requiring mechanical ventilation (part a), significant pain (part b), coma (part c), delirium (part d) and requiring physical constraints (part e). Confidence bands represent the probability of the outcomes and the $95 \% \mathrm{Cl}$, adjusted for baseline ICU admission characteristics and daily covariates. The relationships between the proportion of elements performed and each outcome was significant $(P<0.0001)$ for all outcomes. Below $60-80 \%$ bundle compliance, there was minimal effect on outcomes. Adapted with permission from REF. ${ }^{266}$, Pun, B. T. et al. Caring for critically ill patients with the ABCDEF bundle: results of the ICU liberation collaborative in over 15,000 adults. Crit. Care Med. 47(1), 3-14 https://journals.lww.com/ccmjournal/pages/ default.aspx.

\section{Multidomain treatment of delirium}

Treatment in general settings. The multidomain treatment approach has long been advocated in guidelines and textbooks but, strikingly, only three randomized controlled trials testing its efficacy have been published ${ }^{241}$, with none of these studies showing an overall significant treatment effect. One study of 174 older general medical patients involved a comprehensive, tailored intervention comprising detailed geriatrics assessment, physiotherapy, assistance with orientation, nutritional support and use of atypical antipsychotic drugs if psychotic symptoms were present and cholinesterase inhibitors in patients with significant cognitive impairment ${ }^{271}$. Although this study did not find an overall effect on the primary end points of mortality or institutionalization, the intervention did result in more rapid improvement in delirium and cognitive functioning than in the control group who received usual care. Given the lack of studies and the lack of definitive evidence of the effectiveness of multidomain treatment, recommendations regarding general delirium treatment are based on expert consensus rather than on trial evidence.

Multidomain delirium treatment largely involves interventions that do not include pharmacological therapy other than that used to treat the assumed underlying causes (for example, antibiotics for infections). Antipsychotic agents or other drugs are not recommended as a routine part of the specific treatment for delirium but may be considered if there is intractable distress for which other approaches have been unsuccessful.

As with other aspects of delirium care, evidence is lacking about optimal treatment implementation. However, there is growing interest in quality improvement approaches, which seem to show promise. For example, a combination of staff education and use of the Think, Investigate, Manage, Engage (TIME) bundle ${ }^{241}$, which targets delirium treatment actions in the first 2 hours following diagnosis, led to improvements in the assessment of delirium causes and exacerbating factors $^{272}$. Furthermore, educational interventions can lead to improvements in the process of care; a systematic review of 42 studies involving educational interventions found that $90 \%$ of these studies demonstrated a beneficial effect on one or more measures, some of which were related to the delivery of delirium treatment ${ }^{273}$. Although definitive evidence is lacking, these reviews suggest that educational interventions may be an effective approach to improving implementation that should be evaluated in larger trials.

Treatment in the ICU. Several studies have examined the effectiveness of multidomain delirium treatment in the ICU, focusing predominantly on the ABCDEF bundle (as described above) (TABLE 1).

\section{Pharmacological treatment of delirium}

Treatment in non-ICU settings. In contrast to the very small number of studies examining multidomain approaches for the treatment of delirium, multiple studies have examined drugs as a standalone treatment for delirium. The delirium syndrome, rather than particular features of delirium, such as agitation or psychosis, 
has been the main eligibility criterion for inclusion in these studies; that is, delirium has been considered a single entity in randomized controlled trials of drug treatments. The rationale is that particular neurochemical abnormalities, such as excessive dopaminergic transmission or reduced cholinergic transmission, are assumed to be present in delirium and thus the syndrome is responsive to drugs that act on these abnormalities. However, this theoretical basis is questionable given the likely involvement of multiple neurobiological mechanisms in delirium pathogenesis, as described above.

Antipsychotic agents are the most-studied class of drugs for delirium treatment. A 2018 Cochrane review of antipsychotic agents for the treatment of delirium in non-ICU patients reported findings of 9 trials involving 727 participants ${ }^{274}$. Data were generally of poor quality, with antipsychotic agents having no effect on delirium severity, symptom resolution or mortality. Data on duration, length of stay, discharge destination or QOL were lacking, and reporting of adverse effects was absent or poor. Similarly, a 2019 review of 19 studies (some of which included patients in the ICU) found no effect of antipsychotic agents on delirium duration or severity or length of stay ${ }^{275}$. Antipsychotic drugs and benzodiazepines are widely used for the treatment of delirium in palliative care. A 2020 Cochrane review of four trials testing drug treatment for delirium in terminally ill adults ${ }^{276}$ found that the efficacy of drug therapy was unclear owing to the mostly low to very low quality of evidence in these studies; however, there was some evidence of adverse effects of haloperidol and risperidone.

Cholinesterase inhibitors have also been suggested as a treatment for delirium but the current evidence suggests that these drugs are not effective ${ }^{277}$. A 2018 Cochrane systematic review of studies in non-ICU settings identified only one eligible trial including only

\section{Table 1 | Delirium risk factors targeted by the ABCDEF bundle}

\begin{tabular}{|lll|}
$\begin{array}{l}\text { ABCDEF } \\
\text { bundle } \\
\text { component }^{267}\end{array}$ & Approach & Delirium risk factor \\
\hline A & $\begin{array}{l}\text { Assess, prevent and manage } \\
\text { pain }\end{array}$ & $\begin{array}{l}\text { Undertreated pain; over-sedation } \\
\text { caused by analgesics }\end{array}$ \\
\hline B & $\begin{array}{l}\text { Both spontaneous awakening } \\
\text { trials and spontaneous } \\
\text { breathing trials }\end{array}$ & $\begin{array}{l}\text { Over-sedation; mechanical } \\
\text { ventilation }\end{array}$ \\
\hline C & $\begin{array}{l}\text { Choice of sedation and } \\
\text { analgesia }\end{array}$ & $\begin{array}{l}\text { Over-sedation caused by } \\
\text { analgesics; over-sedation } \\
\text { caused by sedatives; exposure to } \\
\text { deliriogenic sedatives (for example, } \\
\text { benzodiazepines) }\end{array}$ \\
\hline D & $\begin{array}{l}\text { Delirium: assess, prevent and } \\
\text { manage }\end{array}$ & $\begin{array}{l}\text { Disordered sleep-wake cycle; } \\
\text { vision and hearing impairment; } \\
\text { other unrecognized delirium risk } \\
\text { factors }\end{array}$ \\
\hline E & Early mobility and exercise & Immobility \\
\hline F & $\begin{array}{l}\text { Family engagement and } \\
\text { empowerment }\end{array}$ & \begin{tabular}{l} 
Disorientation \\
\hline
\end{tabular}
\end{tabular}

Information compiled from REF. ${ }^{267}$.
15 patients, so there was insufficient evidence of either efficacy or adverse effects ${ }^{278}$. Melatonin and the melatonin receptor agonist ramelteon have also been evaluated as a treatment for delirium (specifically, disruption of the sleep-wake cycle), but no consistent evidence supporting the use of these agents in clinical practice has emerged to date. A small study evaluating the a2-adrenergic receptor agonist clonidine showed that the intervention was safe but the study was underpowered and did not show an effect on delirium ${ }^{279}$. A study of dexmedetomidine as a rescue therapy (when haloperidol failed) for agitation secondary to delirium in critically ill non-ventilated patients ${ }^{280}$ showed that dexmedetomidine had a better effectiveness, safety and cost-benefit profile than haloperidol ${ }^{281}$. In conclusion, the current consensus is that drugs should not be used as a treatment for delirium as a syndrome. Studies examining the use of drugs as a specific treatment for distressing psychotic or affective disturbances in the context of delirium are lacking. However, expert consensus does support a limited role for drugs for the treatment of intractable distress in patients with delirium when other measures have been ineffective ${ }^{241}$.

Treatment in ICU settings. Although beneficial, nonpharmacological management is not always effective in preventing or treating delirium and therefore clinicians often attempt to manage delirium with pharmacological agents. The efficacy of antipsychotic drugs, which are most commonly used to manage delirium in the $\mathrm{ICU}^{281}$, has been evaluated in several randomized trials. A feasibility study in 103 mechanically ventilated patients with or without delirium who were randomly assigned to haloperidol, ziprasidone or placebo treatment ${ }^{282}$ found no difference in outcomes between the treatment groups. Another study compared haloperidol and placebo in 142 mechanically ventilated ICU patients who were delirious or at high risk for delirium and found that the number of days alive without delirium or coma was similar in the two treatment groups, although the occurrence of agitation was lower in the haloperidol group $^{160}$. Based on these data, the Society of Critical Care Medicine (SCCM) PADIS guidelines made the following recommendation: "We suggest not routinely using haloperidol [or] an atypical antipsychotic ... to treat delirium (conditional recommendation, low quality of evidence)"262.

After the latest SCCM PADIS guidelines were released in 2018, the Modifying the Impact of Neuropsychological Dysfunction-USA (MIND-USA) study ${ }^{159}$, the largest randomized trial to examine antipsychotic drugs for the treatment of delirium during critical illness, was published. MIND-USA compared haloperidol, ziprasidone and placebo in 566 ICU patients with delirium and found no significant treatment effects on primary outcomes, including the number of days alive and without delirium or coma (FIG. 7), or on multiple secondary outcomes, including duration of delirium, hypoactive delirium or hyperactive delirium.

Furthermore, a 2019 systematic review of 16 randomized controlled trials and 10 observational studies found no differences in outcomes (such as delirium 
a Days alive without delirium or coma

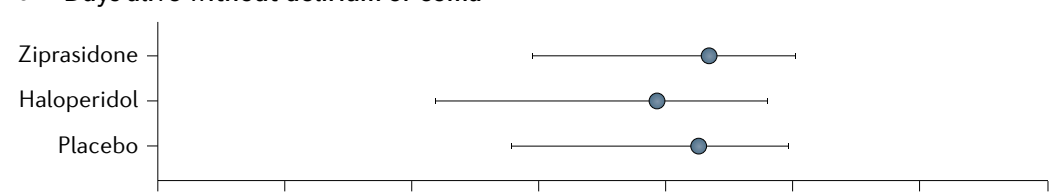

b Days with delirium

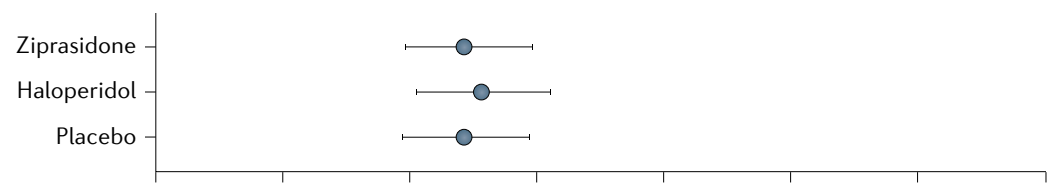

c Days with coma

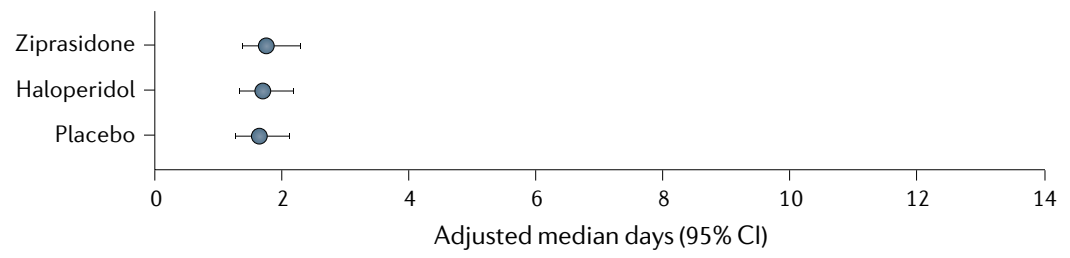

Fig. 7 | Antipsychotic drugs are ineffective in delirium treatment. In the MIND-USA trial ${ }^{159}$, the efficacy of the antipsychotic drugs haloperidol and ziprasidone was compared with placebo for the treatment of delirium in critically ill patients. There was no significant association between study drug (the atypical antipsychotic drug ziprasidone, the typical antipsychotic drug haloperidol or placebo) and days free from delirium or coma (part a), days with delirium (part b) or days with coma (part c). From REF. ${ }^{159}$, The New England Journal of Medicine, Girard, T. D. et al. Haloperidol and ziprasidone for treatment of delirium in critical illness. 379, 2506-2516, Copyright @ (2018) Massachusetts Medical Society. Reprinted with permission.

severity, sedation status, delirium duration, hospital length of stay, cognitive functioning or mortality) between haloperidol and second-generation antipsychotic drugs versus placebo ${ }^{283}$.

Only one placebo-controlled trial found that an antipsychotic agent may be efficacious as a treatment for delirium $^{284}$. In this trial, 36 ICU patients with delirium who were receiving haloperidol were randomly assigned to treatment with the atypical antipsychotic drug quetiapine or placebo, with quetiapine treatment resulting in a shorter time to first resolution of delirium than placebo. A larger confirmatory trial is needed before quetiapine can be recommended as a routine treatment for delirium in the ICU.

Three separate, multicentre randomized trials ${ }^{285-287}$ found that mechanically ventilated ICU patients who are sedated with the centrally acting $\alpha_{2}$-adrenergic receptor agonist dexmedetomidine experience less delirium than those sedated with GABAergic agents, such as benzodiazepines or propofol (FIG. 8). In a placebo-controlled trial to assess whether dexmedetomidine was effective in treating agitated delirium that was preventing extubation in 74 mechanically ventilated ICU patients ${ }^{288}$, delirium resolved 16 hours (40\%) earlier and extubation occurred earlier (median of 17 hours) among patients treated with dexmedetomidine than in those who received placebo. The SCCM PADIS guidelines therefore recommended "using dexmedetomidine for delirium in mechanically ventilated adults where agitation is precluding weaning/ extubation (conditional recommendation, low quality of evidence)"262.
A Cochrane review of pharmacological interventions for the treatment of delirium in critically ill adults included 9 trials and 727 participants from medical, surgical and palliative care services ${ }^{274}$. This review found that, compared with treatment with non-antipsychotic drugs, antipsychotic drugs did not reduce delirium severity (standard mean difference $-1.08,95 \%$ CI -2.55 to 0.39 ); very low quality of evidence), had no effect on resolution of delirium symptoms and did not alter mortality ${ }^{274}$. Furthermore, there was no difference in response between typical and atypical antipsychotic drugs (standard mean difference $-0.17,95 \% \mathrm{CI}-0.37$ to 0.02 ; low quality of evidence) $)^{274}$. Owing to insufficient data, the association of antipsychotic medication exposure with altered duration of delirium or other relevant patient outcomes could not be assessed.

\section{COVID-19 considerations}

Current evidence suggests that the ABCDEF bundle is effective in shortening the duration of delirium ${ }^{266,289,290}$. In light of this evidence, the SCCM made the following recommendation in the 2018 SCCM PADIS Guidelines ${ }^{262}$ : "We suggest using a multicomponent, non-pharmacological intervention that is focused on (but not limited to) reducing modifiable risk factors for delirium, improving cognition, and optimizing sleep, mobility, hearing, and vision in critically ill adults (conditional recommendation, low quality of evidence)." This evidence was graded as low quality in the guidelines because of the lack of randomized trials.

Despite known best practices, in the era of COVID-19, some have speculated that rational and irrational fears of health-care providers may affect usual care, as they may experience a conflict between serving others and maintaining personal well-being ${ }^{291}$. Uncertainty regarding the clinical course of COVID-19 and shifts in health-care provider attitudes and routine behaviour may hinder adherence to the ABCDEF bundle ${ }^{292}$. Reduced adherence may result from changes to the critical care hierarchy, priorities, and ICU team composition and from substantial shortages of personal protective equipment, which cause a reduced and/or restricted physical bedside presence, favouring an increased depth of sedation and use of neuromuscular blockade that exacerbates drug shortages and requires prolonged use of limited ventilator resources ${ }^{292}$. Early studies in COVID-19 cohorts suggest that the use of benzodiazepines for sedation in the ICU is becoming more widespread, with $86 \%$ of patients receiving midazolam and a $65 \%$ prevalence of delirium in one study of a French cohort of 58 patients with COVID-19 (REF. ${ }^{293}$ ). The exact reasons why patients with COVID-19 seem to develop a severe delirium phenotype is unclear but potential reasons include direct central nervous system invasion, induction of central nervous system inflammatory mediators, secondary effects of other organ system failure, effect of sedative strategies, prolonged mechanical ventilation time, immobilization and other needed environmental factors with unfortunate consequences, including social isolation and quarantine without family ${ }^{294}$.

Older adults, especially those residing in care homes, are particularly vulnerable to the isolation practices and 
deviations from standard of care during the COVID-19 pandemic, yet, despite this increased vulnerability, neither mental status changes nor delirium screening are part of COVID-19 assessment ${ }^{295}$. In light of this, some have advocated for a collaborative approach to delirium prevention and management with a doubling down on adherence to established care guidelines, which will benefit not only current but also future patients, especially when resources are scarce ${ }^{296}$.

\section{Quality of life}

Delirium is strongly associated with multiple adverse outcomes. These outcomes may be short-term effects, such as falls, aspiration pneumonia, distress and other events occurring during delirium or in the days following delirium ${ }^{2,297,298}$, or increased short-term mortality ${ }^{27,240,248,299}$. Evidence also exists for an association between delirium and worse long-term outcomes, such as increased 1-year mortality and disability ${ }^{300,301}$. Whether specific components of the delirium syndrome are better at predicting poor outcomes than others remains unclear. For example, in a systematic review, acutely reduced arousal (above the level of coma), which is virtually diagnostic of delirium ${ }^{198}$, was associated with a sixfold increase in 30-day hospital mortality ${ }^{302}$. Another study found that the duration of hypoactive delirium but not that of hyperactive delirium was associated with worse long-term cognitive function among critically ill patients experiencing delirium ${ }^{303}$. The potential influence of delirium aetiology, such as medications, infection or other causes, on outcomes has been explored in a small number of studies, with mixed findings ${ }^{51,81}$. However, these adverse outcomes all have a major role in a patient's QOL.

Patients with acute and critical illness suffer from reduced QOL as a consequence of their illnesses ${ }^{304-306}$,

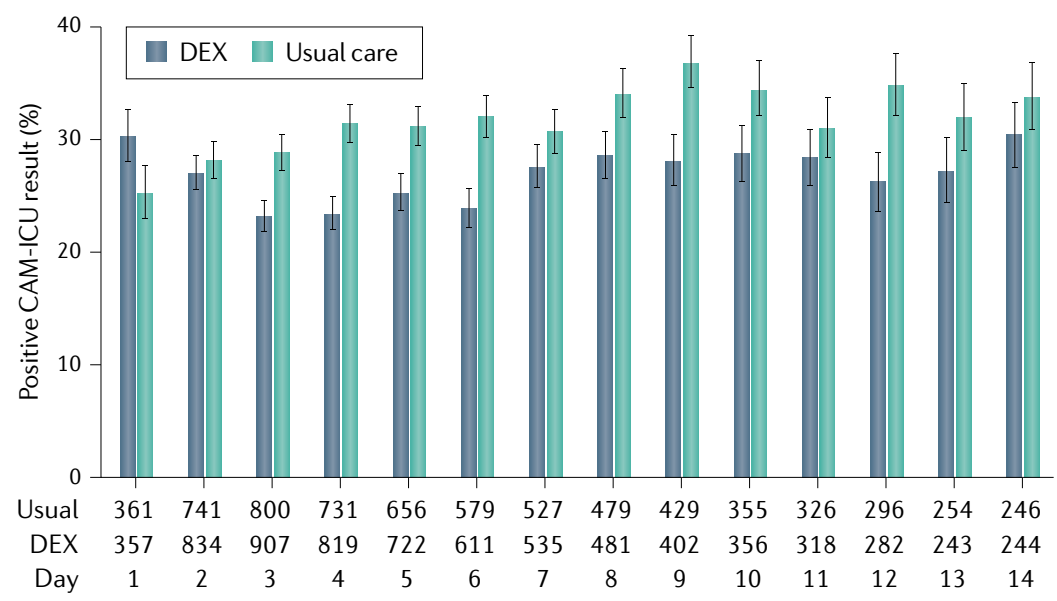

Fig. 8 | Proportion of delirious patients according to sedation regimen (SPICE III trial). Intensive care unit (ICU) patients with a sedation level at Richmond Agitation Sedation Scale (RASS) of -2 or greater were assessed with the Confusion Assessment Method-Intensive Care Unit (CAM-ICU) tool ${ }^{287}$. There were more patients with a positive CAM-ICU in the first 24 hours among ICU patients receiving dexmedetomidine (DEX), an $\alpha_{2}$-adrenergic receptor agonist, compared with usual care sedation regimen (mainly the GABAergic agents propofol or midazolam) as directed by the treating physician. CAM-ICU positivity declined significantly faster over the subsequent treatment days in patients receiving DEX than in those on the usual care regimen (overall $P=0.01$ ). and delirium is an important risk factor owing to persistent cognitive dysfunction, functional disability and worsened mental health (FIG. 9). Although not all critically ill patients experience delirium, those with delirium during admission to a medical or surgical ICU reported lower QOL (using the Medical Outcomes Study Short-Form General Health Survey (SF-36) questionnaire) after discharge than those without delirium $^{307,308}$. Similar reductions in QOL also occurred in recipients of haematopoietic stem cell transplantation or patients with intracerebral haemorrhage ${ }^{309,310}$. However, not all studies have shown a reduced QOL following delirium ${ }^{311,312}$. QOL is multidimensional, including physical, cognitive and psychological domains as well as non-medical domains, such as interpersonal relationships and employment status ${ }^{313-315}$. Instruments routinely used to assess QOL following delirium, such as the SF-36 questionnaire ${ }^{316,317}$ and its derivatives ${ }^{318,319}$, may have variable sensitivity in identifying small but clinically relevant QOL differences ${ }^{320}$. Overall, reduced QOL following delirium is attributable to its role in the subsequent development of neurocognitive dysfunction and worsened mental health.

Impairments in cognitive function are significant contributors to life satisfaction and QOL following acute illness ${ }^{321}$. Population studies have found that patients without dementia at baseline who experience an episode of delirium have a far higher risk of future dementia than those who do not experience delirium. For example, a population study found an almost ninefold increased risk of dementia in adults $\geq 85$ years of age ${ }^{77}$ and delirium was linked with worse cognitive functioning at 1 year in a critical care population ${ }^{3}$. Similarly, in older adults undergoing hip surgery, postoperative delirium was strongly associated with postoperative neurocognitive dysfunction in the form of cognitive impairment and memory decline $\mathrm{e}^{322}$. Furthermore, episodes of delirium may also accelerate decline in people with dementia ${ }^{323}$. For patients with pre-existing Alzheimer disease, the development of delirium while hospitalized is strongly associated with further cognitive decline, institutionalization and mortality ${ }^{324}$. In addition, delirium has been implicated in the development of long-term cognitive impairment (LTCI; a form of acquired dementia) in multiple patient cohorts ${ }^{3,325}$, with estimates as high as two-thirds of patients having substantial impairment at 12 months after an episode of delirium ${ }^{3,326,327}$ (BOX 1). The duration of delirium in ICU patients but not total sedative exposure is associated with a risk of $\mathrm{LTCI}^{3}$. LTCI has been associated with reduced QOL following critical illness ${ }^{328-330}$ or major surgery ${ }^{331}$ and in patients who are long-term transplant survivors ${ }^{332}$. LTCI is also associated with reduced employment at 12 months after discharge from the $\mathrm{ICU}^{333}$ and with employment status after critical illness, which is an important contributor to QOL $^{334}$. Reduced employment is an added stressor and burden for those who experience delirium and LTCI, leading to loss of independence and affecting important non-medical domains of QOL.

Delirium has also been associated with an increased risk of subsequent depression, anxiety and post-traumatic stress disorder ${ }^{335,336}$ and these psychological morbidities 


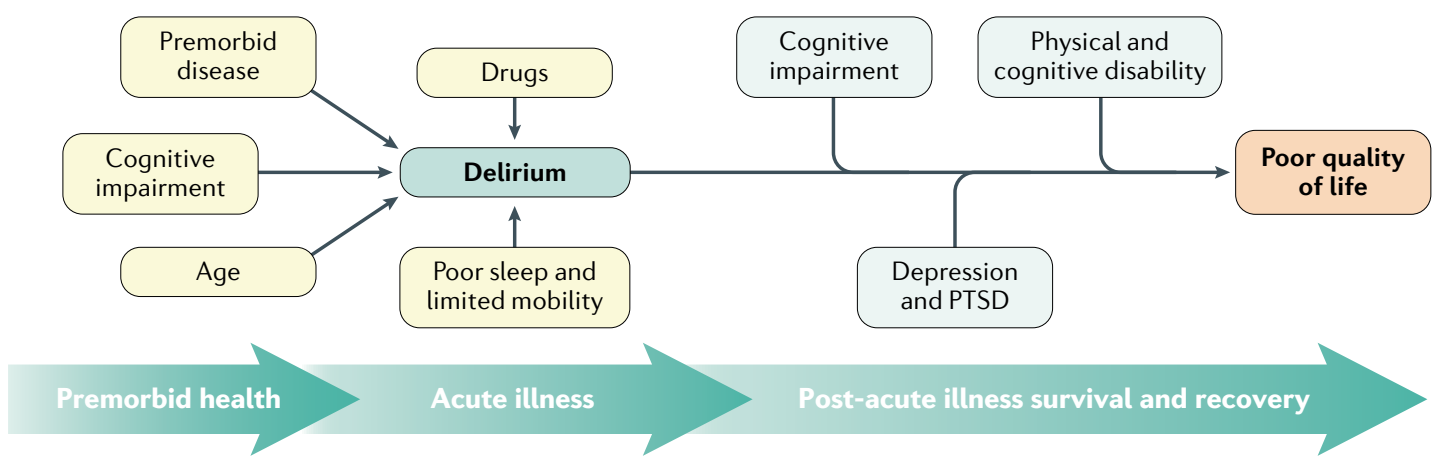

Fig. 9 | Relationship between delirium and post-ICU quality of life. With critical illness as the backdrop, this schematic depicts the association between delirium during an intensive care unit (ICU) stay and downstream impairments that lead to poor quality of life. Multiple potential pre-existing and precipitating risk factors can lead to delirium during critical illness. The development and duration of delirium increase the risk of cognitive, psychiatric and physical impairments, which accumulate to reduce a patient's quality of life. PTSD, post-traumatic stress disorder.

are associated with a reduced $\mathrm{QOL}^{337}$. In survivors of acute lung injury, depression is associated with lower life satisfaction ${ }^{338}$ and post-traumatic stress disorder is associated with worse self-reported QOL $^{339}$. In addition, poor executive function after critical illness, a cardinal feature of LTCI, is correlated with depression and worse mental health-related QOL in ICU survivors ${ }^{340}$. Both cognitive function and mental health are integral components in a patient's $\mathrm{QOL}^{341}$, with both identified as important patient-centred outcomes by survivors of critical illness and their families ${ }^{342}$. Delirium affects both cognitive function and mental health, impairing QOL and functional recovery of patients after their illness.

The long-lasting effects of delirium reach beyond the confines of the brain. Delirium is associated with functional decline and worse disability in acutely hospitalized older adults ${ }^{343}$ and, for older patients admitted with hip fracture, postoperative delirium is strongly associated with future functional decline $\mathrm{e}^{322}$. Among critically ill patients admitted to a medical ICU, the development and duration of delirium was a robust predictor of subsequent worse disability among survivors in the year following critical illness ${ }^{344}$. The inability to return to normal life owing to a decline in functional status and worsening disability following delirium is an important factor in a patient's QOL and remains a major burden faced by patients and caregivers.

Few studies have evaluated methods to improve QOL following delirium. A multimodal geriatric intervention was tested in acutely ill older patients with delirium and resulted in improved QOL without increasing overall health-care costs, with health-care use in the following year for both groups costing approximately US $\$ 22,000$ $\left(\right.$ REF $\left.^{305}\right)$. However, there remains a dearth of knowledge of the modifiable risk factors for reduced QOL following delirium and a paucity of studies evaluating interventions to improve QOL. The substantial, sometimes permanent impairments in QOL that result from delirium necessitate future research that, in addition to prevention, should focus on discovering potential interventions to improve QOL and address the long-term consequences of delirium, including LTCI and comorbid psychiatric disease.

\section{Outlook}

Over the past few decades, great progress has been made in understanding the epidemiology of delirium, including the demographic and clinical risk factors, and in the development of various clinical screening tools to detect delirium across medical and surgical settings. Furthermore, our understanding of the prevention, treatment and clinical effects of delirium (that is, morbidities and mortality) has improved.

\section{Delirium categorization}

What remains less well understood is whether delirium is a singular condition that should be studied regardless of phenotype or aetiology or is really a grouping of various types of deliria that are better studied as distinct physiological conditions.

Delirium research may benefit from further meaningful categorization as a one-size-fits-all approach may not be the best tactic moving forwards ${ }^{345}$. Just as other brain disorders have been subtyped into various domains, such as phenotype (in mood disorders), neuropathological findings (in dementias) or aetiology (in strokes), some groups have categorized delirium on the basis of various features, such as the predominant motor activity subtype (hypoactive, hyperactive or mixed) $)^{346}$ or the aetiology, including hypoxia, sepsis, sedative exposure or metabolic dysfunction (for example, renal or hepatic dysfunction $)^{81}$. Both of these schemas have been useful for prognostication, with hypoactive delirium being associated with worse outcomes, including higher mortality, longer lengths of stay, increased number of falls and institutionalization as well as a lower $\mathrm{QOL}^{76,346-352}$. Delirium associated with sedatives, sepsis and hypoxia has been correlated with worse cognitive functioning at 1 year after the delirium event ${ }^{81}$. Despite advances in exploring pharmacological agents for the prevention and treatment of delirium, current identification systems do not allow for aetiological heterogeneity (that is, splitting) and are therefore subject to bias towards the null hypothesis (that is, that multiple delirium subtypes exist). Just as 'splitting' delirium into subtypes may be useful in exploring the pathophysiology, prevention and treatment of delirium, it may also be equally advantageous to 'lump' 
together delirium subtypes in certain settings to increase advocacy for and awareness of the syndrome.

\section{Management challenges}

The failure of pharmacological treatment studies may be related to the heterogeneous nature of delirium. Perhaps a pharmacological intervention that works for one patient with delirium does not work for another as there may be distinct physiological processes involved that contribute to the final manifestation of delirium. Given that delirium represents a form of acute brain dysfunction that poses a potential risk to all persons worldwide, the need to fully understand the aetiologies and pathophysiology of delirium, to potentially modify existing risk factors and to improve treatment modalities remains a high priority. The neuropathology cascade that leads from delirium to new-onset dementia remains poorly understood and future research should explore this important pathway with the utmost priority.

Unravelling distinct mechanistic routes that lead to delirium should remain an important priority of the field. It seems likely that some forms of delirium, such as those triggered by the administration or withdrawal of particular drugs, might trigger a neurochemical imbalance that is sufficient to produce a delirium episode but which might just as easily be reversed by normalizing these imbalances. By contrast, insults that produce inflammatory or hypoxic damage to the brain are more likely to contribute to long-term outcomes. Therefore, defining mechanisms of acute brain dysfunction that also drives brain injury in animal models should be pursued to drive the identification of plausible inflammatory, metabolic or neurochemical targets for interventions to minimize or prevent acute brain injury during acute illness.

\section{Implementation challenges}

Major challenges remain in the implementation of effective detection, prevention and treatment methods in mainstream clinical practice. Undoubtedly, these challenges are due to the generally poor coverage of delirium in the education and training of medical practitioners and nurses ${ }^{249}$ as well as to the consequent low levels of awareness and skill and lack of appropriate attitudes with respect to 'ownership' of delirium care as one of the most common and serious acute medical conditions ${ }^{14,250}$. Another key issue is the lack of clinical implementation studies ${ }^{353}$. Many tools and validation studies exist but few studies have examined their implementation $^{212,218,252}$. Such studies should examine both the uptake rates of tools and compare their delirium detection rates to locally measured reference standards. Another priority is the design and testing of multidomain delirium treatment processes in general settings, as currently only three randomized controlled trials have tested the advised treatment process across guidelines and position statements. This extraordinary gap in the evidence is surprising given the high prevalence of delirium in older hospitalized patients. The effectiveness of multicomponent protocols in preventing incident delirium is well established but, again, there is a lack of studies examining effective implementation in routine practice.

Published online: 12 November 2020
1. Adamis, D., Treloar, A., Martin, F. C. \& Macdonald, A. J. A brief review of the history of delirium as a mental disorder. Hist. Psychiatry 18, 459-469 (2007).

2. Williams, S. T., Dhesi, J. K. \& Partridge, J. S. L. Distress in delirium: causes, assessment and management. Eur. Geriatr. Med. 11, 63-70 (2020).

3. Pandharipande, P. P. et al. Long-term cognitive impairment after critical illness. N. Engl. J. Med. 369 , 1306-1316 (2013).

This prospective longitudinal cohort study demonstrated that critically ill patients are at risk of LTCI after critical illness, that this new LTCI can persist at 3 and 12 months follow-up, and that it is associated with duration of delirium.

4. American Psychiatric Association. Diagnostic and statistical manual of mental disorders, 5th edn (DSM-5) (American Psychiatric Association Publishing, 2013)

5. Cole, M. G. et al. Partial and no recovery from delirium in older hospitalized adults: frequency and baseline risk factors. J. Am. Geriatr. Soc. 63, 2340-2348 (2015).

6. Ouimet, S. et al. Subsyndromal delirium in the ICU: evidence for a disease spectrum. Intensive Care Med. 33, 1007-1013 (2007).

7. Cole, M., McCusker, J., Dendukuri, N. \& Han, L. The prognostic significance of subsyndromal delirium in elderly medical inpatients. J. Am. Geriatr. Soc. $\mathbf{5 1}$ 754-760 (2003)

8. Slooter, A. J. C. et al. Updated nomenclature of delirium and acute encephalopathy: statement of ten societies. Intensive Care Med. 46, 1020-1022 (2020).

A joint position paper of ten international societies on the near complete segregation of the literature on delirium from that on encephalopathy, with recommendations to separate the underlying brain pathological state, namely encephalopathy, from the manifest clinical features, namely delirium
9. Casey, P. et al. Hospital discharge data under-reports delirium occurrence: results from a point prevalence survey of delirium in a major Australian health service. Intern. Med. J. 49, 338-344 (2019).

10. Oldham, M. A. \& Holloway, R. G. Delirium disorder: Integrating delirium and acute encephalopathy. Neurology 95, 173-178 (2020).

11. Ely, E. W. et al. The impact of delirium in the intensive care unit on hospital length of stay. Intensive Care Med. 27, 1892-1900 (2001).

12. Teodorczuk, A. \& MacLullich, A. New waves of delirium understanding. Int. J. Geriatr. Psychiatry 33 1417-1419 (2018).

13. Lipowski, Z. J. Transient cognitive disorders (delirium, acute confusional states) in the elderly. Am. J. Psychiatry 140, 1426-1436 (1983).

14. Khachaturian, A. S. et al. International drive to illuminate delirium: a developing public health blueprint for action. Alzheimers Dement. 16, 711-725 (2020)

15. Davis, D. H. et al. The epidemiology of delirium: challenges and opportunities for population studies. Am. J. Geriatr. Psychiatry 21, 1173-1189 (2013).

16. Gibb, K. et al. The consistent burden in published estimates of delirium occurrence in medical inpatients over four decades: a systematic review and meta-analysis study. Age Ageing 49, 352-360 (2020).

17. Marcantonio, E. R. Delirium in hospitalized older adults. N. Engl. J. Med. 377, 1456-1466 (2017).

18. Smith, T. O. et al. Factors predicting incidence of postoperative delirium in older people following hip fracture surgery: a systematic review and meta-analysis. Int. $J$. Geriatr. Psychiatry 32, 386-396 (2017).

19. Watt, J. et al. Identifying older adults at risk of delirium following elective surgery: a systematic review and meta-analysis. J. Gen. Intern. Med. 33, 500-509 (2018).

20. Greaves, D. et al. Cognitive outcomes following coronary artery bypass grafting: a systematic review and meta-analysis of 91,829 patients. Int. J. Cardiol. 289, 43-49 (2019).
21. Abawi, M. et al. Postoperative delirium in individuals undergoing transcatheter aortic valve replacement: a systematic review and meta-analysis. J. Am. Geriatr. Soc. 66, 2417-2424 (2018).

22. Shaw, R. C., Walker, G., Elliott, E. \& Quinn, T. J. Occurrence rate of delirium in acute stroke settings: systematic review and meta-analysis. Stroke $\mathbf{5 0}$, 3028-3036 (2019).

23. Watt, C. L. et al. The incidence and prevalence of delirium across palliative care settings: a systematic review. Palliat. Med. 33, 865-877 (2019).

24. Hosie, A., Davidson, P. M., Agar, M., Sanderson, C. R. $\&$ Phillips, J. Delirium prevalence, incidence, and implications for screening in specialist palliative care inpatient settings: a systematic review. Palliat. Med. 27, 486-498 (2013)

25. Morichi, V. et al. A point prevalence study of delirium in Italian nursing homes. Dement. Geriatr. Cogn. Disord. 46, 27-41 (2018).

26. Andrew, M. K., Freter, S. H. \& Rockwood, K. Prevalence and outcomes of delirium in community and non-acute care settings in people without dementia: a report from the Canadian study of health and aging. BMC Med. 4 , 15 (2006).

27. Inouye, S. K., Westendorp, R. G. \& Saczynski, J. S Delirium in elderly people. Lancet 383, 911-922 (2014).

28. Krewulak, K. D. Stelfox, H. T. Leigh J. P. Ely, E. W. \& Fiest, K. M. Incidence and prevalence of delirium subtypes in an adult ICU: a systematic review and meta-analysis. Crit. Care Med. 46, 2029-2035 (2018).

29. Almeida, I. C et al. The impact of acute brain dysfunction in the outcomes of mechanically ventilated cancer patients. PLOS ONE 9, e85332 (2014).

30. Janssen, N. J. et al. On the utility of diagnostic instruments for pediatric delirium in critical illness: an evaluation of the pediatric anesthesia emergence delirium scale, the delirium rating scale 88 , and the delirium rating scale-revised $\mathrm{R}-98$. Intensive Care Med. 37, 1331-1337 (2011). 
31. Larsen, G. Y., Donaldson, A. E., Parker, H. B. $\&$ Grant, M. J. Preventable harm occurring to critically ill children. Pediatr. Crit. Care Med. 8, 331-336 (2007)

32. Schieveld, J. N. et al. Pediatric delirium in critical illness phenomenology, clinical correlates and treatment response in 40 cases in the pediatric intensive care unit. Intensive Care Med. 33, 1033-1040 (2007).

33. Silver, G. et al. Detecting pediatric delirium development of a rapid observational assessment tool. Intensive Care Med. 38, 1025-1031 (2012).

34. Smith, H. A. et al. Diagnosing delirium in critically ill children: validity and reliability of the pediatric confusion assessment method for the intensive care unit. Crit. Care Med. 39, 150-157 (2011).

35. Traube, C. et al. Cornell assessment of pediatric delirium: a valid, rapid, observational tool for screening delirium in the PICU* Crit. Care Med. 42, 656-663 (2014).

36. Smith, H. A. et al. The preschool confusion assessment method for the ICU: valid and reliable delirium monitoring for critically III infants and children. Crit. Care Med. 44, 592-600 (2016).

37. Gross, A. L. et al. Delirium and long-term cognitive trajectory among persons with dementia. Arch. Intern. Med. 172, 1324-1331 (2012)

38. Silver, G. et al. Pediatric delirium and associated risk factors: a single-center prospective observational study. Pediatr. Crit. Care Med. 16, 303-309 (2015).

39. Smith, P. J., Attix, D. K., Weldon, B. C., Greene, N. H. \& Monk, T. G. Executive function and depression as independent risk factors for postoperative delirium. Anesthesiology 110, 781-787 (2009).

40. Wilson, K., Broadhurst, C., Diver, M., Jackson, M $\&$ Mottram, P. Plasma insulin growth factor-1 and incident delirium in older people. Int. J. Geriatr. Psychiatry 20, 154-159 (2005)

41. Velayati, A., Vahdat Shariatpanahi, M., Shahbazi, E. \& Vahdat Shariatpanahi, Z. Association between preoperative nutritional status and postoperative delirium in individuals with coronary artery bypass graft surgery: a prospective cohort study. Nutrition 66, 227-232 (2019)

42. Sanford, A. M. \& Flaherty, J. H. Do nutrients play a role in delirium? Curr. Opin. Clin. Nutr. Metab. Care 17, 45-50 (2014).

43. Persico, I. et al. Frailty and delirium in older adults: a systematic review and meta-analysis of the literature. J. Am. Geriatr. Soc. 66, 2022-2030 (2018).

44. Jung, P. et al. The impact of frailty on postoperative delirium in cardiac surgery patients. J. Thorac. Cardiovasc. Surg. 149, 869-875.e1-2 (2015).

45. Davis, D. H. et al. Worsening cognitive impairment and neurodegenerative pathology progressively increase risk for delirium. Am. J. Geriatr. Psychiatry 23, 403-415 (2015)

This paper represents the clearest demonstration that progressive cognitive decline is a progressively increasing risk factor for delirium and also demonstrates, in mice, that this decline is correlated with increasing synaptic loss and can precede frank neurodegeneration. The study also validates the first animal model of delirium superimposed on dementia.

46. Nitchingham, A., Kumar, V., Shenkin, S., Ferguson, K. J. \& Caplan, G. A. A systematic review of neuroimaging in delirium: predictors, correlates and consequences. Int. J. Geriatr. Psychiatry 33, 1458-1478 (2018).

47. Ferguson, K. J. \& MacLullich, A. M. J. in Brain disorders in Critical Illness (eds Stevens, R. D., Sharshar, T. \& Ely, E. W.) (Cambridge University Press, 2013).

48. McCoy, T. H. Jr. Hart, K., Pellegrini, A. \& Perlis, R. H. Genome-wide association identifies a novel locus for delirium risk. Neurobiol. Aging 68, 160.e9-160.e14 (2018)

49. Adamis, D., Meagher, D., Williams, J., Mulligan, O. \& McCarthy, G. A systematic review and meta-analysis of the association between the apolipoprotein E genotype and delirium. Psychiatr. Genet. 26, 53-59 (2016)

50. Laurila, J. V., Laakkonen, M. L., Laurila, J. V., Timo, S. E. $\&$ Reijo, T. S. Predisposing and precipitating factors for delirium in a frail geriatric population. J. Psychosom. Res. 65, 249-254 (2008).

51. Cirbus, J. et al. Delirium etiology subtypes and their effect on six-month function and cognition in older emergency department patients. Int. Psychogeriatr 31, 267-276 (2019).

52. Clegg, A. \& Young, J. B. Which medications to avoid in people at risk of delirium: a systematic review. Age Ageing 40, 23-29 (2011).

53. Sampson, E. L., West, E. \& Fischer, T. Pain and delirium mechanisms, assessment, management. Eur. Geriatr. Med. 11, 45-52 (2020).
54. Van Rompaey, B. et al. Risk factors for delirium in intensive care patients: a prospective cohort study. Crit. Care 13, R77 (2009)

55. Shehabi, Y. et al. Delirium duration and mortality in lightly sedated, mechanically ventilated intensive care patients. Crit. Care Med. 38, 2311-2318 (2010).

56. Ely, E. W. et al. Delirium in mechanically ventilated patients: validity and reliability of the confusion assessment method for the intensive care unit (CAM-ICU). JAMA 286, 2703-2710 (2001).

57. Pandharipande, P. et al. Prevalence and risk factors for development of delirium in surgical and trauma intensive care unit patients. J. Trauma 65, 34-41 (2008).

58. Sharma, A., Malhotra, S., Grover, S. \& Jindal, S. K. Incidence, prevalence, risk factor and outcome of delirium in intensive care unit: a study from India. Gen. Hosp. Psychiatry 34, 639-646 (2012).

59. Tsuruta, R. et al. Prevalence and associated factors for delirium in critically ill patients at a Japanese intensive care unit. Gen. Hosp. Psychiatry 32, 607-611 (2010)

60. Lindroth, $\mathrm{H}$. et al. Systematic review of prediction models for delirium in the older adult inpatient. BMJ Open 8, e019223 (2018)

61. Rudolph, J. L. et al. Derivation and validation of a preoperative prediction rule for delirium after cardiac surgery. Circulation 119, 229 (2009).

62. O'Keeffe, S. \& Lavan, J. Predicting delirium in elderly patients: development and validation of a riskstratification model. Age Ageing 25, 317-321 (1996).

63. Fisher, B. W. \& Flowerdew, G. A simple model for predicting postoperative delirium in older patients undergoing elective orthopedic surgery. J. Am. Geriatr. Soc. 43, 175-178 (1995)

64. Kalisvaart, K. J. et al. Risk factors and prediction of postoperative delirium in elderly hip-surgery patients: Implementation and validation of a medical risk factor model. J. Am. Geriatr. Soc. 54, 817-822 (2006).

65. Böhner, H. et al. Predicting delirium after vascula surgery: a model based on pre-and intraoperative data. Ann. Surg. 238, 149 (2003).

66. Kennedy, M. et al. Delirium risk prediction, healthcare use and mortality of elderly adults in the emergency department. J. Am. Geriatr. Soc. 62, 462-469 (2014)

67. Wassenaar, A. et al. Multinational development and validation of an early prediction model for delirium in ICU patients. Intensive Care Med. 41, 1048-1056 (2015).

68. Van den Boogaard, M. et al. Development and validation of PRE-DELIRIC (PREdiction of DELIRium in ICu patients) delirium prediction model for intensive care patients: observational multicentre study. $B M J$ 344, e420 (2012)

69. Linkaite, G., Riauka, M., Buneviciute, I. \& Vosylius, S. Evaluation of PRE-DELIRIC (PREdiction of DELIRium in ICu patients) delirium prediction model for the patients in the intensive care unit. Acta Med. Litu. 25 14-22 (2018)

70. Paton, L., Elliott, S. \& Chohan, S. Utility of the PRE-DELIRIC delirium prediction model in a Scottish ICU cohort. J. Intensive Care Soc. 17, 202-206 (2016).

71. Sosa, F. A. et al. Assessment of delirium using the PRE-DELIRIC model in an intensive care unit in Argentina. Rev. Bras. Ter. Intensiva 30, 50-56 (2018).

72. Rudberg, M. A., Pompei, P., Foreman, M. D., Ross, R. E. $\&$ Cassel, C. K. The natural history of delirium in older hospitalized patients: a syndrome of heterogeneity. Age Ageing 26, 169-174 (1997)

73. Cole, M. G., Ciampi, A., Belzile, E. \& Zhong, L. Persistent delirium in older hospital patients: a systematic review of frequency and prognosis. Age Ageing 38, 19-26 (2009).

74. Meagher, D., Adamis, D., Trzepacz, P. \& Leonard, M. Features of subsyndromal and persistent delirium. Br. J. Psychiatry 200, 37-44 (2012).

75. Witlox, J. et al. Delirium in elderly patients and the risk of postdischarge mortality, institutionalization, and dementia: a meta-analysis. JAMA 304, 443-451 (2010).

This meta-analysis provides evidence that, in elderly patients, delirium is associated with poor outcomes (mortality, institutionalization and dementia), independent of important confounders.

76. Jackson, T. A., Wilson, D., Richardson, S. \& Lord, J. M. Predicting outcome in older hospital patients with delirium: a systematic literature review. Int. J. Geriatr. Psychiatry 31, 392-399 (2016)

77. Davis, D. H. et al. Delirium is a strong risk factor for dementia in the oldest-old: a population-based cohort study. Brain 135, 2809-2816 (2012).

78. Goldberg, T. E. et al. Association of delirium with long term cognitive decline: a meta-analysis. JAMA Neurol. https://doi.org/10.1001/jamaneurol.2020.2273 (2020).
79. Engel, G. L. \& Romano, J. Delirium II Reversibility of the electroencephalogram with experimental procedures. Arch. Neuro Psychiatr. 51, 378-392 (1944).

80. Itil, T. \& Fink, M. Anticholinergic drug-induced delirium: experimental modification, quantitative EEC and behavioral correlations. J. Nerv. Ment. Dis. 143, 492-507 (1966).

81. Girard, T. D. et al. Clinical phenotypes of delirium during critical illness and severity of subsequent long-term cognitive impairment: a prospective cohort study. Lancet Respir. Med. 6, 213-222 (2018).

82. Inouye, S. K. \& Charpentier, P. A. Precipitating factors for delirium in hospitalized elderly persons. Predictive model and interrelationship with baseline vulnerability. JAMA 275, 852-857 (1996).

83. Tijms, B. M. et al. Alzheimer's disease: connecting findings from graph theoretical studies of brain networks. Neurobiol. Aging 34, 2023-2036 (2013).

84. Cunningham, C., Wilcockson, D. C., Campion, S. Lunnon, K. \& Perry, V. H. Central and systemic endotoxin challenges exacerbate the local inflammatory response and increase neuronal death during chronic neurodegeneration. J. Neurosci. 25, 9275-9284 (2005).

85. Hennessy, E., Griffin, E. W. \& Cunningham, C. Astrocytes are primed by chronic neurodegeneration to produce exaggerated chemokine and cell infiltration responses to acute stimulation with the cytokines IL-1 beta and TNF-alpha. J. Neurosci. 35, 8411-8422 (2015).

86. Hasel, P. et al. Neurons and neuronal activity control gene expression in astrocytes to regulate their development and metabolism. Nat. Commun. 8 , 15132 (2017).

87. Sweeney, M. D., Kisler, K., Montagne, A., Toga, A. W. $\&$ Zlokovic, B. V. The role of brain vasculature in neurodegenerative disorders. Nat. Neurosci. 21 1318-1331 (2018).

88. Yang, A. C. et al. Physiological blood-brain transport is impaired with age by a shift in transcytosis. Nature 583, 425-430 (2020).

89. Alagiakrishnan, K. \& Wiens, C. A. An approach to drug induced delirium in the elderly. Postgrad. Med. J. 80, 388-393 (2004)

90. Engel, G. L. \& Romano, J. Delirium, a syndrome of cerebral insufficiency. J. Chronic Dis. 9, 260-277 (1959).

This perspective piece gathers many key observations on clinical and volunteer neurophysiology studies detailing the relationship between EEG and delirium symptoms as well as making a compelling case for the role of disturbed brain energy metabolism as a driver of delirium.

91. Taccone, F. S. et al. Cerebral microcirculation is impaired during sepsis: an experimental study. Crit. Care 14 R140 (2010).

92. Yokota, H., Ogawa, S., Kurokawa, A. \& Yamamoto, Y. Regional cerebral blood flow in delirium patients. Psychiatry Clin. Neurosci. 57, 337-339 (2003).

93. Pfister, D. et al. Cerebral perfusion in sepsisassociated delirium. Crit. Care 12, R63 (2008)

94. Caplan, G. A. et al. Cerebrospinal fluid in long-lasting delirium compared with Alzheimer's dementia. J. Gerontol. A Biol. Sci. Med. Sci. 65, 1130-1136 (2010).

95. Kealy, J. et al. Acute inflammation alters brain energy metabolism in mice and humans: role in suppressed spontaneous activity, impaired cognition, and delirium. J. Neurosci. 40, 5681-5696 (2020).

96. Bendahan, N., Neal, O., Ross-White, A., Muscedere, J. $\&$ Boyd, J. G. Relationship between near-infrared spectroscopy-derived cerebral oxygenation and delirium in critically ill patients: a systematic review. J. Intensive Care Med. 34, 514-520 (2019).

97. Rosengarten, B. et al. Microcirculatory dysfunction in the brain precedes changes in evoked potentials in endotoxin-induced sepsis syndrome in rats. Cerebrovasc. Dis. 23, 140-147 (2007).

98. Polito, A. et al. Pattern of brain injury in the acute setting of human septic shock. Crit. Care 17, R204 (2013).

99. Reddy, P. H. \& Beal, M. F. Amyloid beta, mitochondrial dysfunction and synaptic damage: implications for cognitive decline in aging and Alzheimer's disease. Trends Mol. Med. 14, 45-53 (2008)

100. Shehab, N. et al. US emergency department visits for outpatient adverse drug events, 2013-2014. JAMA 316, 2115-2125 (2016)

101. Sejling, A. S. et al. Hypoglycemia-associated changes in the electroencephalogram in patients with type 1 diabetes and normal hypoglycemia awareness or unawareness. Diabetes 64, 1760-1769 (2015). 
102. Gugger, J. J., Geocadin, R. G. \& Kaplan, P. W. A multimodal approach using somatosensory evoked potentials for prognostication in hypoglycemic encephalopathy. Clin. Neurophysiol. Pract. 4, 194-197 (2019)

103. Sonneville, R. et al. Potentially modifiable factors contributing to sepsis-associated encephalopathy. Intensive Care Med. 43, 1075-1084 (2017).

104. Thorell, A., Efendic, S., Gutniak, M., Haggmark, T. \& Ljungqvist, O. Insulin resistance after abdomina surgery. Br. J. Surg. 81, 59-63 (1994).

105. Virkamaki, A., Puhakainen, I., Koivisto, V. A , Vuorinen-Markkola, H. \& Yki-Jarvinen, H. Mechanisms of hepatic and peripheral insulin resistance during acute infections in humans. J. Clin. Endocrinol. Metab. 74, 673-679 (1992)

106. Meltzer, C. C. et al. Regional hypometabolism in Alzheimer's disease as measured by positron emission tomography after correction for effects of partial volume averaging. Neurology 47, 454-461 (1996).

107. Holscher, C. Insulin signalling impairment in the brain as a risk factor in Alzheimer's disease. Front. Aging Neurosci. 11, 88 (2019)

108. Semmler, A. et al. Sepsis causes neuroinflammation and concomitant decrease of cerebral metabolism. J. Neuroinflammation $\mathbf{5}, 38$ (2008).

109. Haggstrom, L. R., Nelson, J. A., Wegner, E. A. \& Caplan, G. A. 2-(18)F-fluoro-2-deoxyglucose positron emission tomography in delirium. J. Cereb. Blood Flow Metab. 37, 3556-3567 (2017).

110. Zimmer, E. R. et al. $\left[{ }^{18} \mathrm{~F}\right] \mathrm{FDG}$ PET signal is driven by astroglial glutamate transport. Nat. Neurosci. $\mathbf{2 0}$, 393-395 (2017)

111. Maclullich, A. M., Ferguson, K. J., Miller, T., de Rooij, S. E. \& Cunningham, C. Unravelling the pathophysiology of delirium: a focus on the role of aberrant stress responses. J. Psychosom. Res. 65 229-238 (2008)

112. Yu, M. et al. HMGB1 signals through toll-like receptor (TLR) 4 and TLR2. Shock 26, 174-179 (2006).

113. Deiner, S. et al. Human plasma biomarker responses to inhalational general anaesthesia without surgery. Br. J. Anaesth. https://doi.org/10.1016/ j.bja.2020.04.085 (2020)

114. Dantzer, R., O'Connor, J. C., Freund, G. G., Johnson, R. W. \& Kelley, K. W. From inflammation to sickness and depression: when the immune system subjugates the brain. Nat. Rev. Neurosci. 9, 46-56 (2008).

115. Cunningham, C. Microglia and neurodegeneration: the role of systemic inflammation. Glia 61, 71-90 (2013).

116. Subramaniyan, S. \& Terrando, N. Neuroinflammation and perioperative neurocognitive disorders. Anesth. Analg. 128, 781-788 (2019).

117. Banks, W. A., Farr, S. A. \& Morley, J. E. Entry of bloodborne cytokines into the central nervous system: effects on cognitive processes. Neuroimmunomodulation 10 319-327 (2002)

118. Cerejeira, J., Firmino, H., Vaz-Serra, A. \& Mukaetova-Ladinska, E. B. The neuroinflammatory hypothesis of delirium. Acta Neuropathol. 119 737-754 (2010)

119. van Gool, W. A., van de Beek, D. \& Eikelenboom, P. Systemic infection and delirium: when cytokines and acetylcholine collide. Lancet 375, 773-775 (2010).

120. van den Boogaard, M. et al. Biomarkers associated with delirium in critically ill patients and their relation with long-term subjective cognitive dysfunction; indications for different pathways governing delirium in inflamed and noninflamed patients. Crit. Care 15, R297 (2011).

121. Vasunilashorn, S. M. et al. High C-reactive protein predicts delirium incidence, duration, and feature severity after major noncardiac surgery. J. Am. Geriatr. Soc. 65, e 109-e116 (2017)

122. Vasunilashorn, S. M. et al. Cytokines and postoperative delirium in older patients undergoing major elective surgery. J. Gerontol. A Biol. Sci. Med. Sci. 70 1289-1295 (2015)

123. Henjum, K. et al. CSF sTREM2 in delirium-relation to Alzheimer's disease CSF biomarkers A $\beta 42$, t-tau and p-tau. J. Neuroinflammation 15, 304 (2018).

124. van Munster, B. C. et al. Neuroinflammation in delirium: a postmortem case-control study. Rejuvenation Res. 14, 615-622 (2011).

125. Feng, X. et al. Microglia mediate postoperative hippocampal inflammation and cognitive decline in mice. JCl Insight 2, e91229 (2017).

126. Cibelli, M. et al. Role of interleukin- 1 beta in postoperative cognitive dysfunction. Ann. Neurol. 68, 360-368 (2010)
127. Frank, M. G. et al. IL-1RA blocks E. coli-induced suppression of Arc and long-term memory in aged F344xBN F1 rats. Brain Behav. Immun. 24, 254-262 (2010).

128. Pugh, C. R. et al. Role of interleukin- 1 beta in impairment of contextual fear conditioning caused by social isolation. Behav. Brain Res. 106, 109-118 (1999).

129. Skelly, D. T. et al. Acute transient cognitive dysfunction and acute brain injury induced by systemic inflammation occur by dissociable IL-1-dependent mechanisms. Mol. Psychiatry 24, 1533-1548 (2019).

This paper provides a partial mechanistic basis for acute lipopolysaccharide-induced delirium-like deficits selectively in mice with prior neurodegeneration. The data suggest that acute cognitive deficits and acute brain injury may be dissociable, driven by systemic and centrally produced IL-1 $\beta$, respectively.

130. Cape, E. et al. Cerebrospinal fluid markers of neuroinflammation in delirium: a role for interleukin-1 beta in delirium after hip fracture. J. Psychosom. Res. 77 219-225 (2014).

131. Serantes, R. et al. Interleukin-1 beta enhances GABAA receptor cell-surface expression by a phosphatidylinositol 3-kinase/Akt pathway: relevance to sepsis-associated encephalopathy. J. Biol. Chem. 281, 14632-14643 (2006)

132. Liu, X. et al. Cell-type-specific interleukin 1 receptor 1 signaling in the brain regulates distinct neuroimmune activities. Immunity 50, 764-766 (2019).

133. Lopez-Rodriguez, A. B. et al. Microglial and astrocyte priming in the APP/PS1 model of Alzheimer's disease: increased vulnerability to acute inflammation and cognitive deficits. Preprint at bioRxiv https://doi.org 10.1101/344218 (2018)

134. Andonegui, G. et al. Targeting inflammatory monocytes in sepsis-associated encephalopathy and long-term cognitive impairment. JCl Insight https://doi.org/ 10.1172/jci.insight.99364 (2018).

135. Vacas, S., Degos, V., Tracey, K. J. \& Maze, M. High-mobility group Box 1 protein initiates postoperative cognitive decline by engaging bone marrow-derived macrophages. Anesthesiology 120, 1160-1167 (2014).

136. Waltl, I. et al. Macrophage depletion by liposomeencapsulated clodronate suppresses seizures but not hippocampal damage after acute viral encephalitis. Neurobiol. Dis. 110, 192-205 (2018)

137. MacLullich, A. M. et al. Cerebrospinal fluid interleukin-8 levels are higher in people with hip fracture with perioperative delirium than in controls. J. Am. Geriatr. Soc. 59, 1151-1153 (2011).

138. van Munster, B. C. et al. Time-course of cytokines during delirium in elderly patients with hip fractures. J. Am. Geriatr. Soc. 56, 1704-1709 (2008).

139. Hall, R. J. et al. CSF biomarkers in delirium: a systematic review. Int. J. Geriatr. Psychiatry 33, 1479-1500 (2018).

140. Skrede, K., Wyller, T. B., Watne, L. O., Seljeflot, I. \& Juliebo, V. Is there a role for monocyte chemoattractan protein-1 in delirium? Novel observations in elderly hip fracture patients. BMC Res. Notes 8, 186 (2015).

141. Campbell, S. J. et al. Sickness behaviour is induced by a peripheral CXC-chemokine also expressed in multiple sclerosis and EAE. Brain Behav. Immun. 24 738-746 (2010)

142. Le Thuc, O. et al. Central CCL2 signaling onto MCH neurons mediates metabolic and behavioral adaptation to inflammation. EMBO Rep. 17, 1738-1752 (2016).

143. Marciniak, E. et al. The chemokine MIP-1 alpha/CCL3 impairs mouse hippocampal synaptic transmission, plasticity and memory. Sci. Rep. 5, 15862 (2015).

144. Varatharaj, A. \& Galea, I. The blood-brain barrier in systemic inflammation. Brain Behav. Immun. 60 , 1-12 (2017)

145. Hov, K. R. et al. Blood-cerebrospinal fluid barrier integrity in delirium determined by Q-Albumin. Dement. Geriatr. Cognit. Disord. 41, 192-198 (2016).

146. The Lancet Haematology. COVID-19 coagulopathy: an evolving story. Lancet Haematol. 7, e425 (2020).

147. Forsberg, A. et al. The immune response of the human brain to abdominal surgery. Ann. Neurol. 81 572-582 (2017)

148. Ebert, U. \& Kirch, W. Scopolamine model of dementia: electroencephalogram findings and cognitive performance. Eur. J. Clin. Invest. 28, 944-949 (1998).

149. Trzepacz, P. T. Anticholinergic model for delirium Semin. Clin. Neuropsychiatry 1, 294-303 (1996).

150. Tune, L. E. Serum anticholinergic activity levels and delirium in the elderly. Semin. Clin. Neuropsychiatry 5, 149-153 (2000).
151. Carnahan, R. M., Lund, B. C., Perry, P. J., Pollock, B. C. $\&$ Culp, K. R. The anticholinergic drug scale as a measure of drug-related anticholinergic burden: associations with serum anticholinergic activity. J. Clin. Pharmacol. 46, 1481-1486 (2006).

152. Liptzin, B., Laki, A., Garb, J. L., Fingeroth, R. \& Krushell, R. Donepezil in the prevention and treatment of post-surgical delirium. Am. J. Geriatr. Psychiatry 13, 1100-1106 (2005)

153. Marcantonio, E. R., Palihnich, K., Appleton, P. $\&$ Davis, R. B. Pilot randomized trial of donepezil hydrochloride for delirium after hip fracture. J. Am . Geriatr. Soc. 59 (Suppl. 2), S282-S288 (2011).

154. van Eijk, M. M. et al. Effect of rivastigmine as an adjunct to usual care with haloperidol on duration of delirium and mortality in critically ill patients: a multicentre, double-blind, placebo-controlled randomised trial. Lancet 376, 1829-1837 (2010)

155. Field, R. H., Gossen, A. \& Cunningham, C. Prior pathology in the basal forebrain cholinergic system predisposes to inflammation induced working memory deficits: reconciling inflammatory and cholinergic hypotheses of delirium. J. Neurosci. 32, 6288-6294 (2012).

156. McKeith, I. et al. Efficacy of rivastigmine in dementia with Lewy bodies: a randomised, double-blind, placebo-controlled international study Lancet 356 , 2031-2036 (2000).

157. Roy, R., Niccolini, F., Pagano, G. \& Politis, M. Cholinergic imaging in dementia spectrum disorders. Eur. J. NuCl. Med. Mol. Imaging 43, 1376-1386 (2016).

158. Agar, M. R. et al. Efficacy of oral risperidone haloperidol, or placebo for symptoms of delirium among patients in palliative care: a randomized clinical trial. JAMA Intern. Med. 177, 34-42 (2017).

159. Girard, T. D. et al. Haloperidol and ziprasidone for treatment of delirium in critical illness. N. Engl. J. Med. 379, 2506-2516 (2018).

This randomized, double-blind, placebo-controlled trial (MIND-USA) evaluated haloperidol, ziprasidone or placebo for treating delirium in critically ill patients with respiratory failure or shock, finding no effect of these antipsychotic drugs on days alive without delirium or coma, or duration of delirium or coma.

160. Page, V. J. et al. Effect of intravenous haloperidol on the duration of delirium and coma in critically ill patients (Hope-ICU): a randomised, double-blind, placebo-controlled trial. Lancet Respir. Med. 1, 515-523 (2013)

161. van den Boogaard, M. et al. Effect of haloperidol on survival among critically ill adults with a high risk of delirium: the REDUCE randomized clinical trial. JAMA 319, 680-690 (2018). In the multisite, randomized, double-blinded, placebo-controlled REDUCE trial comparing prophylactic haloperidol with placebo for delirium prevention in critically ill adults, haloperidol did not improve survival at $\mathbf{2 8}$ days; thus, prophylactic haloperidol is not recommended for reducing mortality in critically ill adults.

162. Gainetdinov, R. R., Jones, S. R. \& Caron, M. G. Functional hyperdopaminergia in dopamine transporter knock-out mice. Biol. Psychiatry 46, 303-311 (1999).

163. Scammell, T. E., Jackson, A. C., Franks, N. P., Wisden, W. \& Dauvilliers, Y. Histamine: neural circuits and new medications. Sleep https://doi.org/10.1093/sleep/ zsy 183 (2019).

164. Chazot, P. L., Johnston, L., McAuley, E. \& Bonner, S. Histamine and delirium: current opinion. Front Pharmacol. 10, 299 (2019).

165. Aston-Jones, G \& Cohen, J. D. An integrative theory of locus coeruleus-norepinephrine function: adaptive gain and optimal performance. Annu. Rev. Neurosci. 28, 403-450 (2005)

166. Arnsten, A. F. Stress signalling pathways that impair prefrontal cortex structure and function. Nat. Rev. Neurosci. 10, 410-422 (2009).

167. Matthews, K. L. et al. Noradrenergic changes, aggressive behavior, and cognition in patients with dementia. Biol. Psychiatry 51, 407-416 (2002).

168. Hahn, P. Y. et al. Sustained elevation in circulating catecholamine levels during polymicrobial sepsis. Shock 4, 269-273 (1995).

169. Buhler, H. U., da Prada, M., Haefely, W. \& Picotti, G. B. Plasma adrenaline, noradrenaline and dopamine in man and different animal species. J. Physiol. 276, 311-320 (1978)

170. Deiner, S., Lin, H. M., Bodansky, D., Silverstein, J. $\&$ Sano, M. Do stress markers and anesthetic technique predict delirium in the elderly? Dement Geriatr. Cogn. Disord. 38, 366-374 (2014). 
171. Cursano, S. et al. A CRHR1 antagonist prevents synaptic loss and memory deficits in a trauma-induced delirium-like syndrome. Mol. Psychiatry https://doi.org/ 10.1038/s41380-020-0659-y (2020)

172. Hawley, R. J. et al. Neurochemical correlates of sympathetic activation during severe alcohol withdrawal. Alcohol. Clin. Exp. Res. 18, 1312-1316 (1994).

173. Smith, A. J., Brent, P. J., Henry, D. A. \& Foy, A. Plasma noradrenaline, platelet alpha 2-adrenoceptors, and functional scores during ethanol withdrawal. Alcohol. Clin. Exp. Res. 14, 497-502 (1990)

174. Muzyk, A. J., Fowler, J. A., Norwood, D. K $\S$ Chilipko, A. Role of alpha2-agonists in the treatment of acute alcohol withdrawal. Ann. Pharmacother. 45 649-657 (2011).

175. Maldonado, J. R. Novel algorithms for the prophylaxis and management of alcohol withdrawal syndromes beyond benzodiazepines. Crit. Care Clin. 33 . 559-599 (2017)

176. Skrobik, Y., Duprey, M. S., Hill, N. S. \& Devlin, J. W. Low-dose nocturnal dexmedetomidine prevents ICU delirium. A randomized, placebo-controlled trial. Am. J. Respir. Crit. Care Med. 197, 1147-1156 (2018)

177. Su, X. et al. Dexmedetomidine for prevention of delirium in elderly patients after non-cardiac surgery: a randomised, double-blind, placebo-controlled trial. Lancet 388, 1893-1902 (2016).

178. Liu, X. et al. Dexmedetomidine vs propofol sedation reduces delirium in patients after cardiac surgery: a meta-analysis with trial sequential analysis of randomized controlled trials. J. Crit. Care 38 190-196 (2017)

179. Ventura, R., Alcaro, A. \& Puglisi-Allegra, S. Prefrontal cortical norepinephrine release is critical for morphineinduced reward, reinstatement and dopamine release in the nucleus accumbens. Cereb. Cortex 15, 1877-1886 (2005).

180. Sanders, R. D. Hypothesis for the pathophysiology of delirium: role of baseline brain network connectivity and changes in inhibitory tone. Med. Hypotheses 77. 140-143 (2011).

181. Pandharipande, P. et al. Lorazepam is an independent risk factor for transitioning to delirium in intensive care unit patients. Anesthesiology 104, 21-26 (2006).

182. Zaal, I. J. et al. Benzodiazepine-associated delirium in critically ill adults. Intensive Care Med. 41, 2130-2137 (2015)

183. Vlisides, P. \& Avidan, M. Recent Advances in Preventing and Managing Postoperative Delirium. F1000Res https://doi.org/10.12688/f1000research.16780.1 (2019).

184. Yoshitaka, S., Egi, M., Kanazawa, T., Toda, Y. \& Morita, K. The association of plasma gammaaminobutyric acid concentration with postoperative delirium in critically ill patients. Crit. Care Resusc. 16, 269-273 (2014).

185. Morandi, A. et al. The relationship between delirium duration, white matter integrity, and cognitive impairment in intensive care unit survivors as determined by diffusion tensor imaging: the VISIONS prospective cohort magnetic resonance imaging study. Crit. Care Med. 40, 2182-2189 (2012).

186. Cavallari, M. et al. Neural substrates of vulnerability to postsurgical delirium as revealed by presurgical diffusion MRI. Brain 139, 1282-1294 (2016).

187. Murray, C. et al. Systemic inflammation induces acute working memory deficits in the primed brain: relevance for delirium. Neurobiol. Aging 33 . 603-616.e3 (2012)

188. Peters van Ton, A. M., Verbeek, M. M., Alkema, W., Pickkers, P. \& Abdo, W. F. Downregulation of synapseassociated protein expression and loss of homeostatic microglial control in cerebrospinal fluid of infectious patients with delirium and patients with Alzheimer's disease. Brain Behav. Immun. https://doi.org/10.1016 j.bbi.2020.06.027 (2020).

189. van Montfort, S. J. T. et al. Brain network disintegration as a final common pathway for delirium: a systematic review and qualitative meta-analysis. Neuroimage Clin. 23, 101809 (2019)

190. Stam, C. J. Modern network science of neurological disorders. Nat. Rev. Neurosci. 15, 683-695 (2014).

191. Choi, S. H. et al. Neural network functional connectivity during and after an episode of delirium Am. J. Psychiatry 169, 498-507 (2012)

192. Raichle, M. E. The brain's default mode network. Annu. Rev. Neurosci. 38, 433-447 (2015).

193. Fleischmann, R. et al. Delirium is associated with frequency band specific dysconnectivity in intrinsic connectivity networks: preliminary evidence from a large retrospective pilot case-control study. Pilot. Feasibility Stud. 5, 2 (2019).
194. Numan, T. et al. Functional connectivity and network analysis during hypoactive delirium and recovery from anesthesia. Clin. Neurophysiol. 128, 914-924 (2017)

195. van Montfort, S. J. T. et al. Resting-state fMRI reveals network disintegration during delirium. Neuroimage Clin. 20, 35-41 (2018).

196. Zhang, L. J., Wu, S., Ren, J. \& Lu, G. M. Resting-state functional magnetic resonance imaging in hepatic encephalopathy: current status and perspectives. Metab. Brain Dis. 29, 569-582 (2014).

197. American Psychiatric Association. Diagnostic and Statistical Manual of Mental Disorders 4th edn (American Psychiatric Association, 1994).

198. European Delirium Association \& American Delirium Society. The DSM- 5 criteria, level of arousal and delirium diagnosis: inclusiveness is safer. BMC Med. 12, 141 (2014).

199. Cole, M. G., Dendukuri, N., McCusker, J. \& Han, L. An empirical study of different diagnostic criteria for delirium among elderly medical inpatients. J. Neuropsychiatry Clin. Neurosci. 15, 200-207 (2003).

200. Laurila, J. V., Pitkala, K. H., Strandberg, T. E. $\&$ Tilvis, R. S. The impact of different diagnostic criteria on prevalence rates for delirium. Dement. Geriatr. Cogn. Disord. 16, 156-162 (2003).

201. Trzepacz, P. T. A review of delirium assessment instruments. Gen. Hosp. Psychiatry 16, 397-405 (1994).

202. Tieges, Z., Evans, J. J., Neufeld, K. J. \& MacLullich, A. M. J. The neuropsychology of delirium: advancing the science of delirium assessment. Int. J. Geriatr. Psychiatry 33, 1501-1511 (2018).

203. Network for Investigation of Delirium: Unifying Scientists (NIDUS). Delirium Measurement Info Cards https://deliriumnetwork.org/measurement/ delirium-info-cards/ (2020)

204. van Velthuijsen, E. L. et al. Psychometric properties and feasibility of instruments for the detection of delirium in older hospitalized patients: a systematic review. Int. J. Geriatr. Psychiatry 31, 974-989 (2016)

205. De, J. \& Wand, A. P. Delirium screening: a systematic review of delirium screening tools in hospitalized patients. Gerontologist 55, 1079-1099 (2015).

206. Neufeld, K. J. et al. Delirium diagnosis methodology used in research: a survey-based study. Am. J. Geriatr Psychiatry 22, 1513-1521 (2014)

207. Inouye, S. K. et al. Clarifying confusion: the confusion assessment method. A new method for detection of delirium. Ann. Intern. Med. 113, 941-948 (1990). This study reports the creation and validation of the CAM; a multitude of CAM-based assessment tools for use in specific patient populations have since been validated and used worldwide.

208. Shenkin, S. D. et al. Delirium detection in older acute medical inpatients: a multicentre prospective comparative diagnostic test accuracy study of the 4AT and the confusion assessment method. BMC Med. 17 , 138 (2019).

This STARD-compliant randomized controlled diagnostic trial compared the performance of two of the most widely-used delirium detection tools employed in general settings, the 4AT and the CAM

209. Heinrich, T. W., Kato, H., Emanuel, C. \& Denson, S. Improving the validity of nurse-based delirium screening: a head-to-head comparison of nursing delirium-screening scale and short confusion assessment method. Psychosomatics 60, 172-178 (2019).

210. Hshieh, T. T., Inouye, S. K. \& Oh, E. S. Delirium in the elderly. Clin. Geriatr. Med. 36, 183-199 (2020).

211. Inouye, S. K., Foreman, M. D., Mion, L. C., Katz, K. H. $\&$ Cooney, L. M. Jr. Nurses' recognition of delirium and its symptoms: comparison of nurse and researcher ratings. Arch. Intern. Med. 161, 2467-2473 (2001)

212. Rohatgi, N. et al. Initiative for prevention and early identification of delirium in medical-surgical units: lessons learned in the past five years. Am. J. Med. 132, 1421-1430.e8 (2019).

213. Reynish, E. L. et al. Epidemiology and outcomes of people with dementia, delirium, and unspecified cognitive impairment in the general hospital: prospective cohort study of 10,014 admissions. BMC Med. 15, 140 (2017).

214. Marcantonio, E. R et al. 3D-CAM: derivation and validation of a 3-minute diagnostic interview for CAM-defined delirium: a cross-sectional diagnostic test study. Ann. Intern. Med. 161, 554-561 (2014).

215. Han, J. H. et al. A quick and easy delirium assessment for nonphysician research personnel. Am. J. Emerg. Med. 34, 1031-1036 (2016).

216. Bellelli, G. et al. Validation of the 4AT, a new instrument for rapid delirium screening: a study in 234 hospitalised older people. Age Ageing 43, 496-502 (2014).
217. Tieges, Z. M., et al. Diagnostic accuracy of the 4AT for delirium detection: systematic review and metaanalysis. Preprint at medRxiv https://doi.org 10.1101/2020.06.11.20128280 (2020).

218. Royal College of Physicians. National hip fracture database annual report September 2018. NHFD https://www.nhfd.co.uk/files/2018ReportFiles/ NHFD-2018-Annual-Report-v101.pdf (2018).

219. Maldonado, J. R. et al. A study of the psychometric properties of the "Stanford Proxy Test for Delirium" (S-PTD): a new screening tool for the detection of delirium. Psychosomatics 61, 116-126 (2020).

220. Ely, E. W. et al. Monitoring sedation status over time in ICU patients: reliability and validity of the Richmond agitation-sedation scale (RASS). JAMA 289 2983-2991 (2003).

221. Chester, J. G., Beth Harrington, M., Rudolph, J. L $\&$ VA Delirium Working Group. Serial administration of a modified Richmond agitation and sedation scale for delirium screening. J. Hosp. Med. 7, 450-453 (2012).

222. Royal College of Physicians. National Early Warning Score (NEWS) 2. Standardising the assessment of acute-illness severity in the NHS: additional implementation guidance. $R C P$ https://www.rcplondon ac.uk/file/20716/download (2020).

223. Voyer, P. et al. Recognizing acute delirium as part of your routine [RADAR]: a validation study. BMC Nurs. 14, 19 (2015)

224. Detroyer, E. et al. Detection of delirium in palliative care unit patients: a prospective descriptive study of the delirium observation screening scale administered by bedside nurses. Palliat. Med. 28, 79-86 (2014).

225. Gaudreau, J. D., Gagnon, P., Harel, F., Tremblay, A. $\&$ Roy, M.-A. Fast, systematic, and continuous delirium assessment in hospitalized patients: the nursing delirium screening scale. J. Pain Symptom Manage. 29, 368-375 (2005)

226. Gélinas, C. et al. Delirium assessment tools for use in critically ill adults: a psychometric analysis and systematic review. Crit. Care Nurse 38, 38-49 (2018).

227. Ely, E. W. et al. Evaluation of delirium in critically ill patients: validation of the confusion assessment method for the intensive care unit (CAM-ICU). Crit. Care Med. 29, 1370-1379 (2001)

228. Gusmao-Flores, D., Salluh, J. I., Chalhub, R. A. \& Quarantini, L. C. The confusion assessment method for the intensive care unit (CAM-ICU) and intensive care delirium screening checklist (ICDSC) for the diagnosis of delirium: a systematic review and metaanalysis of clinical studies. Crit. Care 16, R115 (2012)

229. Bergeron, N., Dubois, M.-J., Dumont, M., Dial, S. \& Skrobik, Y. Intensive care delirium screening checklist: evaluation of a new screening tool. Intensive Care Med. 27, 859-864 (2001).

230. Fick, D. M. \& Marcantonio, E. R. In response to "Preliminary development of an ultrabrief two-item bedside test for delirium". J. Hosp. Med. 11, 155 (2016).

231. Lin, H. S. et al. Screening in delirium: a pilot study of two screening tools, the simple query for easy evaluation of consciousness and simple question in delirium. Australas. J. Ageing 34, 259-264 (2015)

232. Trzepacz, P. T. et al. Validation of the delirium rating scale-revised-98: comparison with the delirium rating scale and the cognitive test for delirium. J. Neuropsychiatry Clin. Neurosci. 13, 229-242 (2001).

233. Adamis, D. et al. Reliability of delirium rating scale (DRS) and delirium rating scale-revised-98 (DRS-R98) using variance-based multivariate modelling. J. Psychiatr. Res. 47, 966-971 (2013).

234. Hart, R. P. et al. Validation of a cognitive test for delirium in medical ICU patients. Psychosomatics 37 533-546 (1996)

235. Tieges, Z. et al. A smartphone-based test for the assessment of attention deficits in delirium: A casecontrol diagnostic test accuracy study in older hospitalised patients. PLOS ONE 15, e0227471 (2020).

236. Tang, E. et al. Development and feasibility of a smartphone-based test for the objective detection and monitoring of attention impairments in delirium in the ICU. J. Crit. Care 48, 104-111 (2018)

237. Jones, R. N. et al. Assessment of instruments for measurement of delirium severity: a systematic review. JAMA Intern. Med. 179, 231-239 (2019).

238. Breitbart, W. et al. The memorial delirium assessment scale. J. Pain Symptom Manage. 13, 128-137 (1997).

239. Inouye, S. K. et al. The CAM-S: development and validation of a new scoring system for delirium severity in 2 cohorts. Ann. Intern. Med. 160, 526-533 (2014). 
240. Ely, E. W. et al. Delirium as a predictor of mortality in mechanically ventilated patients in the intensive care unit. JAMA 291, 1753-1762 (2004)

241. Scottish Intercollegiate Guidelines Network (SIGN). SIGN 157. Risk reduction and management of delirium: a national clinical guideline. SIGN https:// www.sign.ac.uk/media/1423/sign 157.pdf (2019).

242. Hijazi, Z., Lange, P., Watson, R. \& Maier, A. B. The use of cerebral imaging for investigating delirium aetiology. Eur. J. Intern. Med. 52, 35-39 (2018).

243. Haggstrom, L., Welschinger, R. \& Caplan, G. A. Functional neuroimaging offers insights into delirium pathophysiology: a systematic review. Australas. J. Ageing 36, 186-192 (2017)

244. Caplan, G. A., Haggstrom, L. R., Nelson, J. A. \& Wegner, E. A. Novel unique pattern of cerebral glucose hypometabolism seen on 2-18 F-Fluoro-2-deoxyglucose positron emission tomography in delirium. Alzheimers Dement. 13, P1533 (2017).

245. Thomas, C. et al. Serum anticholinergic activity and cerebral cholinergic dysfunction: an EEG study in frail elderly with and without delirium. BMC Neurosci. 9, 86 (2008).

246. Van Der Kooi, A. W. et al. Delirium detection using EEG. Chest 147, 94-101 (2015)

247. Fleischmann, R. et al. Diagnostic performance and utility of quantitative EEG analyses in delirium: confirmatory results from a large retrospective casecontrol study. Clin. EEG Neurosci. 50, 111-120 (2019)

248. Geriatric Medicine Research Collaborative. Delirium is prevalent in older hospital inpatients and associated with adverse outcomes: results of a prospective multi-centre study on World Delirium Awareness Day. BMC Med. 17, 229 (2019).

249. Copeland, C. $\&$ Barron, D. T. "Delirium: an essential component in undergraduate training?". Nurse Educ. Today 85, 104211 (2020)

250. Teodorczuk, A., Mukaetova-Ladinska, E., Corbett, S $\&$ Welfare, M. Reconceptualizing models of delirium education: findings of a grounded theory study. Int. Psychogeriatr. 25, 645-655 (2013).

Delirium detection, treatment and preventive strategies remain poorly implemented at scale in clinical practice. This study provides novel and crucia insights into the status of delirium in health-care settings, including the necessity for attitudinal change and ownership of responsibility for delirium care in addition to standard educational strategies.

251. Richardson, S. J., Fisher, J. M. \& Teodorczuk, A The future hospital: a blueprint for effective delirium care. Future Hosp. J. 3, 178-181 (2016).

252. Vardy, E. et al. Use of a digital delirium pathway and quality improvement to improve delirium detection in the emergency department and outcomes in an acute hospital. Age Ageing 49, 672-678 (2020)

253. Dormandy, L., Mufti, S., Higgins, E., Bailey, C. \& Dixon, M. Shifting the focus: a Ol project to improve the management of delirium in patients with hip fracture. Future Healthc. J. 6, 215-219 (2019)

254. Inouye, S. K. et al. A multicomponent intervention to prevent delirium in hospitalized older patients. N. Engl. J. Med. 340, 669-676 (1999).

255. Hshieh, T. T., Yang, T., Gartaganis, S. L., Yue, J. $\&$ Inouye, S. K. Hospital elder life program: systematic review and meta-analysis of effectiveness. $A m$. J. Geriatr. Psychiatry 26, 1015-1033 (2018).

256. National Institute for Health and Care Excellence (NICE) Delirium: prevention, diagnosis and management. NICE https://www.nice.org.uk/guidance/cg 103 (2019).

257. Marcantonio, E. R., Flacker, J. M., Wright, R. J. \& Resnick, N. M. Reducing delirium after hip fracture: a randomized trial. J. Am. Geriatr. Soc. 49, 516-522 (2001).

258. Ludolph, P. et al. Non-pharmacologic multicomponen interventions preventing delirium in hospitalized people. J. Am. Geriatr. Soc. https://doi.org/10.1111/ igs. 16565 (2020)

259. Smith, J. et al. Investigation of ward fidelity to a multicomponent delirium prevention intervention during a multicentre, pragmatic, cluster randomised, controlled feasibility trial. Age Ageing 49, 648-655 (2020).

260. Woodhouse, R. et al. Interventions for preventing delirium in older people in institutional long-term care. Cochrane Database Syst. Rev. 4, CD009537 (2019).

261. Ely, E. W., Siegel, M. D. \& Inouye, S. K. Delirium in the intensive care unit: an under-recognized syndrome of organ dysfunction. Semin. Respir. Crit. Care Med. 22 115-126 (2001)

262. Devlin, J. et al. Clinical practice guidelines for the prevention and management of pain, agitation sedation, delirium, immobility, and sleep disruption in adult patients in the ICU. Crit. Care Med. 46 e825-e873 (2018).

263. Schweickert, W. D. et al. Early physical and occupationa therapy in mechanically ventilated, critically ill patients: a randomised controlled trial. Lancet 373, 1874-1882 (2009).

264. Schaller, S. J. et al. Early, goal-directed mobilisation in the surgical intensive care unit: a randomised controlled trial. Lancet 388, 1377-1388 (2016).

265. Marra, A., Ely, E. W., Pandharipande, P. P. \& Patel, M. B. The ABCDEF bundle in critical care. Crit. Care Clin. 33 . 225-243 (2017)

266. Pun, B. T. et al. Caring for critically ill patients with the ABCDEF bundle: results of the ICU liberation collaborative in over 15,000 adults. Crit. Care Med. 47, 3-14 (2019).

267. Trogrlic ${ }^{-}$Z. et al A systematic review of implementation strategies for assessment, prevention, and management of ICU delirium and their effect on clinical outcomes. Crit. Care 19, 157 (2015).

268. Oh, E. S. et al. Antipsychotics for preventing delirium in hospitalized adults: a systematic review. Ann. Intern. Med. 171, 474-484 (2019)

269. Asleson, D. R. \& Chiu, A. W. Melatonin for delirium prevention in acute medically ill, and perioperative geriatric patients. Aging Med. 3, 132-137 (2020).

270. Siddiqi, N et al Interventions for preventing delirium in hospitalised non-ICU patients. Cochrane Database Syst. Rev. 3, CD005563 (2016). This Cochrane review examined the evidence base on delirium prevention and concluded that moderateto-strong evidence exists that such interventions are effective and should be implemented.

271. Pitkala, K. H., Laurila, J. V., Strandberg, T. E. $\&$ Tilvis, R. S. Multicomponent geriatric intervention for elderly inpatients with delirium: a randomized, controlled trial. J. Gerontol. A Biol. Sci. Med. Sci 61 176-181 (2006).

272. Bauernfreund, Y., Butler, M., Ragavan, S. \& Sampson, E. L. TIME to think about delirium improving detection and management on the acute medical unit. BMJ Open Qual. 7, e000200 (2018)

273. Lee, S. Y. et al. Developing delirium best practice: a systematic review of education interventions for healthcare professionals working in inpatient settings. Eur. Geriatr. Med. 11, 1-32 (2020).

274. Burry, L. et al. Antipsychotics for treatment of delirium in hospitalised non-ICU patients. Cochrane Database Syst. Rev. 6, CD005594 (2018).

275. Neufeld, K. J., Yue, J., Robinson, T. N., Inouye, S. K. $\&$ Needham, D. M. Antipsychotic medication for prevention and treatment of delirium in hospitalized adults: a systematic review and meta-analysis. J. Am . Geriatrics Soc. 64, 705-714 (2016).

276. Finucane, A. M. et al. Drug therapy for delirium in terminally ill adults. Cochrane Database Syst. Rev. 1, CD004770 (2020).

277. Tampi, R R. Tampi, D. J \& Ghori, A K Acetylcholinesterase inhibitors for delirium in older adults. Am. J. Alzheimers Dis. Other Demen. 31 305-310 (2016)

278. Yu, A. et al. Cholinesterase inhibitors for the treatment of delirium in non-ICU settings. Cochrane Database Syst. Rev. 6, CD012494 (2018).

279. Hov, K. R. et al. The Oslo study of clonidine in elderly patients with delirium; LUCID: a randomised placebocontrolled trial. Int. J. Geriatr. Psychiatry 34 974-981 (2019).

280. Carrasco, G. et al. Dexmedetomidine for the treatment of hyperactive delirium refractory to haloperidol in nonintubated ICU patients: a nonrandomized controlled trial. Crit. Care Med. 44, 1295-1306 (2016).

281. Patel, R. P. et al. Delirium and sedation in the intensive care unit: survey of behaviors and attitudes of 1384 healthcare professionals. Crit. Care Med. 37 825-832 (2009).

282. Girard, T. D. et al. Feasibility, efficacy, and safety of antipsychotics for intensive care unit delirium: the MIND randomized, placebo-controlled trial. Crit. Care Med. 38, 428-437 (2010)

283. Nikooie, R. et al. Antipsychotics for treating delirium in hospitalized adults: a systematic review. Ann. Intern. Med. 171, 485-495 (2019).

This systematic review concluded that there is insufficient evidence supporting the routine use of antipsychotic agents for the treatment of delirium

284. Devlin J. W. et al. Efficacy and safety of quetiapine in critically ill patients with delirium: a prospective, multicenter, randomized, double-blind, placebo-controlled pilot study. Crit. Care Med. 38, 419-427 (2010).

285. Pandharipande, P. P. et al. Effect of sedation with dexmedetomidine vs lorazepam on acute brain ysfunction in mechanically ventilated patients. JAMA 298, 2644-2653 (2007)

286. Riker, R. R. et al. Dexmedetomidine vs midazolam for sedation of critically ill patients: a randomized trial. JAMA 301, 489-499 (2009).

287. Shehabi, Y. et al. Early sedation with dexmedetomidin in critically ill ventilated patients. N. Engl. J. Med. 380 2506-2517 (2019).

The SPICE-III trial comparing dexmedetomidine sedation with usual-care regimens (propofol, midazolam or other sedatives) in 4,000 critically ill patients ventilated $>24$ hours; death rate at 90 days was similar for both treatments with more days alive free of delirium and coma, and ventilator free, with more adverse events reported in dexmedetomidinetreated patients.

288. Reade, M. C. et al. Effect of dexmedetomidine added to standard care on ventilator-free time in patients with agitated delirium: a randomized clinical trial. JAMA 315, 1460-1468 (2016).

289. Balas, M. C. et al. Effectiveness and safety of the awakening and breathing coordination, delirium monitoring/management, and early exercise/mobility bundle. Crit. Care Med. 42, 1024-1036 (2014).

290. Barnes-Daly, M. A., Phillips, G. \& Ely, E. W. Improving hospital survival and reducing brain dysfunction at seven california community hospitals: implementing PAD guidelines via the ABCDEF bundle in 6,064 patients. Crit. Care Med. 45, 171-178 (2017)

291. O’Neal, H. R. Jr. Lin, J. C., Devlin, J. W. \& Ely, E. W. Coronavirus disease 2019: harnessing healthy fear via knowledge, attitudes, and behavior. Crit. Care Explor 2, e0149 (2020)

292. Devlin, J. W. et al. Strategies to optimize ICU liberation (A to $\mathrm{F}$ ) bundle performance in critically ill adults with coronavirus disease 2019. Crit. Care Explor. 2, e0139 (2020).

293. Helms, J. et al. Neurologic features in severe SARSCoV-2 infection. N. Engl. J. Med. 382, 2268-2270 (2020).

294. Kotfis, K. et al. COVID-19: ICU delirium management during SARS-CoV-2 pandemic. Crit. Care 24, 176 (2020).

295. O'Hanlon, S. \& Inouye, S. K. Delirium: a missing piece in the COVID-19 pandemic puzzle. Age Ageing 49, 497-498 (2020).

296. LaHue, S. C et al. Collaborative delirium prevention in the age of COVID-19. J. Am. Geriatr. Soc. 68 947-949 (2020)

297. Sillner, A. Y., Holle, C. L. \& Rudolph, J. L. The overlap between falls and delirium in hospitalized older adults: a systematic review. Clin. Geriatr. Med. 35, 221-236 (2019).

298. Dharmarajan, K., Swami, S., Gou, R. Y., Jones, R. N. $\&$ Inouye, S. K. Pathway from delirium to death: potential in-hospital mediators of excess mortality. J. Am. Geriatr. Soc. 65, 1026-1033 (2017).

299. Israni, J., Lesser, A., Kent, T. \& Ko, K. Delirium as a predictor of mortality in US Medicare beneficiaries discharged from the emergency department: a national claims-level analysis up to 12 months. BMJ Open 8, e021258 (2018).

300. Pisani, M. A. et al. Days of delirium are associated with 1-year mortality in an older intensive care unit population. Am. J. Respir. Crit. Care Med. 180 1092-1097 (2009).

301. Altman, M. T. et al. Association of intensive care unit delirium with sleep disturbance and functional disability after critical illness: an observational cohort study. Ann. Intensive Care 8, 63 (2018)

302. Todd, A. et al. Reduced level of arousal and increased mortality in adult acute medical admissions: a systematic review and meta-analysis. BMC Geriatr. 17 283 (2017).

303. Hayhurst, C. J. et al. Association of hypoactive and hyperactive delirium with cognitive function after critical illness. Crit. Care Med. 48, e480-e488 (2020).

304. Herridge, M. S. et al. Functional disability 5 years after acute respiratory distress syndrome. N. Engl. J. Med. 364, 1293-1304 (2011).

305. Pitkala, K. H. et al. Multicomponent geriatric intervention for elderly inpatients with delirium: effects on costs and health-related quality of life. J. Gerontol. A Biol. Sci. Med. Sci. 63, 56-61 (2008).

306. Hshieh, T. T. et al. Trajectory of functional recovery after postoperative delirium in elective surgery. Ann. Surg. 265, 647-653 (2017).

307. Van Rompaey, B. et al. Long term outcome after delirium in the intensive care unit. J. Clin. Nurs. 18, 3349-3357 (2009).

308. Abelha, F. J. et al. Outcome and quality of life in patients with postoperative delirium during an ICU 
stay following major surgery. Crit. Care 17, R257 (2013)

309. Fann, J. R., Alfano, C. M., Roth-Roemer, S., Katon, W. J. \& Syrjala, K. L. Impact of delirium on cognition, distress, and health-related quality of life after hematopoietic stem-cell transplantation. J. Clin. Oncol. 25 1223-1231 (2007)

310. Naidech, A. M. et al. Intracerebral hemorrhage and delirium symptoms. Length of stay, function, and quality of life in a 114-patient cohort. Am. J. Respir. Crit. Care Med. 188, 1331-1337 (2013).

311. van den Boogaard, M. et al. Delirium in critically ill patients: impact on long-term health-related quality of life and cognitive functioning. Crit. Care Med. 40 112-118 (2012)

312. Wolters, A. E. et al. Long-term outcome of delirium during intensive care unit stay in survivors of critical illness: a prospective cohort study. Crit. Care 18, R125 (2014)

313. Gill, T. M. \& Feinstein, A. R. A critical appraisal of the quality of quality-of-life measurements. JAMA $\mathbf{2 7 2}$, 619-626 (1994).

314. Bergner, M. Quality of life, health status, and clinical research. Med. Care 27, S148-S156 (1989).

315. Cella, D. F. Quality of life: concepts and definition. J. Pain Symptom Manage. 9, 186-192 (1994).

316. Ware, J. E. Jr. \& Sherbourne, C. D. The MOS 36-item short-form health survey (SF-36). I. Conceptual framework and item selection. Med. Care 30 , 473-483 (1992)

317. Ware, J. E. SF-36 Physical and Mental Health Summary Scales: A User's Manual Vol. 5 (Health Assessment Lab, New England Medical Center, 1994).

318. Ware, J. Jr. Kosinski, M. \& Keller, S. D. A 12 -item short-form health survey: construction of scales and preliminary tests of reliability and validity. Med. Care 34, 220-233 (1996)

319. Ware, J. E., Kosinski, M. \& Keller, S. D. SF-12: how to Score the SF-12 Physical and Mental Health Summary Scales 3rd edn Vol. 3 (Qualitymetric, 1998).

320. Yang, F., Dawes, P., Leroi, I. \& Gannon, B. Measurement tools of resource use and quality of life in clinical trials for dementia or cognitive impairment interventions: a systematically conducted narrative review. Int. J. Geriatr. Psychiatry 33, e166-e176 (2018).

321. Jackson, J. C., Mitchell, N. \& Hopkins, R. O. Cognitive functioning, mental health, and quality of life in ICU survivors: an overview. Psychiatr. Clin. North. Am. 38 91-104 (2015).

322. Bickel, H., Gradinger, R., Kochs, E. \& Forstl, H. High risk of cognitive and functional decline after postoperative delirium. A three-year prospective study. Dement. Geriatr. Cogn. Disord. 26, 26-31 (2008)

323. Fong, T. G. et al. Delirium accelerates cognitive decline in Alzheimer disease. Neurology 72, 1570-1575 (2009).

324. Fong, T. G. et al. Adverse outcomes after hospitalization and delirium in persons with Alzheimer disease. Ann. Intern. Med. 156, 848-856, W296 (2012).

325. Saczynski, J. S. et al. Cognitive trajectories after postoperative delirium. N. Engl. J. Med. 367, 30-39 (2012).

326. Hopkins, R. O. et al. Neuropsychological sequelae and impaired health status in survivors of severe acute respiratory distress syndrome. Am. J. Respir. Crit. Care Med. 160, 50-56 (1999).

327. Girard, T. D. et al. Delirium as a predictor of long-term cognitive impairment in survivors of critical illness. Crit. Care Med. 38, 1513-1520 (2010).

328. Rothenhausler, H. B., Ehrentraut, S., Stoll, C. Schelling, G. \& Kapfhammer, H. P. The relationship between cognitive performance and employment and health status in long-term survivors of the acute respiratory distress syndrome: results of an exploratory study. Gen. Hosp. Psychiatry 23, 90-96 (2001)

329. Mikkelsen, M. E. et al. Cognitive, mood and quality of life impairments in a select population of ARDS survivors. Respirology 14, 76-82 (2009).

330. Hopkins, R. O. et al. Two-year cognitive, emotional, and quality-of-life outcomes in acute respiratory distress syndrome. Am. J. Respir. Crit. Care Med. 171 340-347 (2005)

331. Phillips-Bute, B. et al. Association of neurocognitive function and quality of life 1 year after coronary artery bypass graft (CABG) surgery. Psychosom. Med. 68 , 369-375 (2006)

332. Lewis, M. B. \& Howdle, P. D. Cognitive dysfunction and health-related quality of life in long-term liver transplant survivors. Liver Transplant. 9, 1145-1148 (2003).

333. Norman, B. C. et al. Employment outcomes after critical illness: an analysis of the bringing to light the risk factors and incidence of neuropsychological dysfunction in ICU survivors cohort. Crit. Care Med. 44, 2003-2009 (2016).

334. Myhren, H., Ekeberg, O. \& Stokland, O. Health-related quality of life and return to work after critical illness in general intensive care unit patients: a 1-year follow-up study. Crit. Care Med. 38, 1554-1561 (2010).

335. Girard, T. D. et al. Risk factors for posttraumatic stress disorder symptoms following critical illness requiring mechanical ventilation: a prospective cohort study. Crit. Care 11, R28 (2007)

336. Jackson, J. C. et al. Depression, post-traumatic stress disorder, and functional disability in survivors of critica illness in the BRAIN-ICU study: a longitudinal cohort study. Lancet Respir. Med. 2, 369-379 (2014).

337. Hopkins, R. O., Weaver, L. K., Chan, K. J. \& Orme, J. F. Jr. Quality of life, emotional, and cognitive function following acute respiratory distress syndrome. J. Int. Neuropsychol. Soc. 10, 1005-1017 (2004).

338. Weinert, C. R., Gross, C. R., Kangas, J. R., Bury, C. L. \& Marinelli, W. A. Health-related quality of life after acute lung injury. Am. J. Respir. Crit. Care Med. 156, 1120-1128 (1997).

339. Kapfhammer, H. P., Rothenhausler, H. B. Krauseneck, T., Stoll, C. \& Schelling, G. Posttraumatic stress disorder and health-related quality of life in long-term survivors of acute respiratory distress syndrome. Am. J. Psychiatry 161, 45-52 (2004).

340. Duggan, M. C. et al. The relationship between executive dysfunction, depression, and mental health-related quality of life in survivors of critical illness: results from the BRAIN-ICU investigation. J. Crit. Care 37, 72-79 (2017).

341. Wilson, I. B. \& Cleary, P. D. Linking clinical variables with health-related quality of life. A conceptual model of patient outcomes. JAMA 273, 59-65 (1995).

342. Dinglas, V. D. et al. Perspectives of survivors, families and researchers on key outcomes for research in acute respiratory failure. Thorax 73, 7-12 (2018).

343. O'Keeffe, S. $\&$ Lavan, J. The prognostic significance of delirium in older hospital patients. J. Am. Geriatr. Soc 45, 174-178 (1997)

344. Brummel, N. E et al. Delirium in the ICU and subsequent long-term disability among survivors of mechanical ventilation. Crit. Care Med. 42, 369-377 (2014).

345. Oldham, M. A., Flaherty, J. H. \& Maldonado, J. R. Refining delirium: a transtheoretical model of delirium disorder with preliminary neurophysiologic subtypes. Am. J. Geriatr. Psychiatry 26, 913-924 (2018).

346. Meagher, D. J. et al. A longitudinal study of motor subtypes in delirium: relationship with other phenomenology, etiology, medication exposure and prognosis. J. Psychosom. Res. 71, 395-403 (2011).

347. Gual, N. et al. Delirium subtypes and associated characteristics in older patients with exacerbation of chronic conditions. Am. J. Geriatr. Psychiatry 26 1204-1212 (2018)

348. Kiely, D. K., Jones, R. N., Bergmann, M. A. \& Marcantonio, E. R. Association between psychomotor activity delirium subtypes and mortality among newly admitted post-acute facility patients. J. Gerontol. A Biol. Sci. Med. Sci. 62, 174-179 (2007).

349. Robinson, T. N., Raeburn, C. D., Tran, Z. V. Brenner, L. A. \& Moss, M. Motor subtypes of postoperative delirium in older adults. Arch. Surg. 146, 295-300 (2011).

350. Bellelli, G., Speciale, S., Barisione, E. \& Trabucchi, M. Delirium subtypes and 1-year mortality among elderly patients discharged from a post-acute rehabilitation facility. J. Gerontol. A Biol. Sci. Med. Sci. 62 , 1182-1183 (2007)

351. Peritogiannis, V., Bolosi, M., Lixouriotis, C. \& Rizos, D. V. Recent insights on prevalence and corelations of hypoactive delirium. Behav. Neurol. 2015, 416792 (2015).

352. Yang, F. M. et al. Phenomenological subtypes of delirium in older persons: patterns, prevalence, and prognosis. Psychosomatics 50, 248-254 (2009).

353. Mudge, A. M. et al. CHERISH (collaboration for hospitalised elders reducing the impact of stays in hospital): protocol for a multi-site improvement program to reduce geriatric syndromes in older inpatients. $B M C$ Geriatr. 17, 11 (2017)

354. Saper, C. B., Scammell, T. E. \& Lu, J. Hypothalamic regulation of sleep and circadian rhythms. Nature 437, 1257-1263 (2005)

355. Halaas, N. B. et al. Neurofilament light in serum and cerebrospinal fluid of hip fracture patients with delirium. Dement. Geriatr. Cogn. Disord. 46 346-357 (2018)

356. Idland, A. V. et al. CSF neurofilament light levels predict hippocampal atrophy in cognitively healthy older adults. Neurobiol. Aging 49, 138-144 (2017).
357. Casey, C. P. et al. Postoperative delirium is associated with increased plasma neurofilament light. Brain 143 47-54 (2019).

358. Semmler, A. et al. Persistent cognitive impairment, hippocampal atrophy and EEG changes in sepsis survivors. J. Neurol. Neurosurg. Psychiatry 84 62-69 (2013).

359. Torvell, M. et al. A single systemic inflammatory insult causes acute motor deficits and accelerates disease progression in a mouse model of human tauopathy. Alzheimers Dement. 5, 579-591 (2019).

\section{Acknowledgements}

J.E.W. acknowledges salary support from the Vanderbilt Clinical and Translational Research Scholars program (1KL2TR002245) and from NIH grants (GM120484 and HL111111). M.F.M. acknowledges training and salary support from the Vanderbilt Clinical and Translational Training Program in Pulmonary Medicine (NIH 5T32HL087738) and research funding from the Vanderbilt University Medical Center Arthur and Lisa Wheeler Critical Care Research Fund. C.C. acknowledges research grants from the NIH (AG050626), WT SRF090907 and ARUK. Y.S. acknowledges research grants from the National Health and Medical Research Council of Australia. T.D.G. acknowledges support from NIH grants (HL135144 and HL143507). A.M.J.M. acknowledges funding from the Medical Research Council and the National Institute for Health Research. E.W.E. discloses additional funding for his time from NIH grant AG027472 and salary support from the Tennessee Valley Healthcare System Geriatric Research Education and Clinical Center (GRECC).

\section{Author contributions}

Introduction (J.E.W., A.J.C.S., A.M.J.M. and E.W.E.); Epidemiology (J.E.W., A.M.J.M. and E.W.E.); Mechanisms/ pathophysiology (C.C. and A.J.C.S.); Diagnosis, screening and prevention (J.E.W., Y.S., A.M.J.M. and E.W.E.); Management (J.E.W., T.D.G., A.M.J.M. and E.W.E.); Quality of life (J.E.W., M.F.M. and E.W.E.); Outlook (J.E.W., A.M.J.M. and E.W.E.); Overview of Primer (J.E.W.). All authors listed above have contributed substantially to the conception or design of the work or to the acquisition, analysis, or interpretation of data for the work and have participated in drafting the work or revising it critically for important intellectual content. Additionally, each author has given their approval to the final version of the manuscript and has agreed to be accountable for all aspects of the work in ensuring that questions related to the accuracy or integrity of any part of the work are appropriately investigated and resolved.

\section{Competing interests}

C.C. acknowledges a current research collaboration with IONIS pharmaceuticals. Y.S. acknowledges unrestricted research grants from Pfizer, Orion Pharma, and Brainstem Biometrics and travel reimbursements and speaker honorarium for educational non-promotional symposia from Pfizer and Orion Pharma. A.J.C.S. is an adviser for Prolira, a start-up company that develops an EEG-based delirium monitor; any (future) profits from EEG-based delirium monitoring will be used for future scientific research only. A.M.J.M. is the main author of the 4AT delirium assessment tool (this tool is free to use and there is no financial interest) and holds patents for computerized delirium assessment tools (any future profits will be used for future scientific research only). E.W.E. received honoraria from Orion and Hospira for continuing medical education activity but does not hold stock or consultant relationships with these companies. The other authors declare no competing interests.

\section{Peer review information}

Nature Reviews Disease Primers thanks K. Neufeld, Y. Skrobik, M. Agar and the other, anonymous, reviewer(s) for their contribution to the peer review of this work.

\section{Publisher's note}

Springer Nature remains neutral with regard to jurisdictional claims in published maps and institutional affiliations.

\section{RELATED LINIKS}

Network for Investigation of Delirium: Unifying Scientists (NIDUS): https://deliriumnetwork.org/

Preserving your brain health during illness or surgery: $\mathrm{GCBH}$ recommendations to prevent and treat delirium: https://www.aarp.org/health/brain-health/

The Critical Illness, Brain Dysfunction and Survivorship (CIBS) Center: https://www.icudelirium.org/cibs-center/

The Hospital Elder Life Program (HELP): https://www. hospitalelderlifeprogram.org

(c) Springer Nature Limited 2020, corrected publication 2020 